\title{
The spinorial geometry of supersymmetric IIB backgrounds
}

\author{
U. Gran ${ }^{1}$, J. Gutowski ${ }^{2}$ and G. Papadopoulos ${ }^{1}$ \\ ${ }^{1}$ Department of Mathematics \\ King's College London \\ Strand \\ London WC2R 2LS, UK \\ 2 Mathematical Institute \\ Oxford University \\ Oxford OX1 3LB, UK
}

\begin{abstract}
We investigate the Killing spinor equations of IIB supergravity for one Killing spinor. We show that there are three types of orbits of $\operatorname{Spin}(9,1)$ in the space of Weyl spinors which give rise to Killing spinors with stability subgroups $\operatorname{Spin}(7) \ltimes$ $\mathbb{R}^{8}, S U(4) \ltimes \mathbb{R}^{8}$ and $G_{2}$. We solve the Killing spinor equations for the $\operatorname{Spin}(7) \ltimes \mathbb{R}^{8}$ and $S U(4) \ltimes \mathbb{R}^{8}$ invariant spinors, give the fluxes in terms of the geometry and determine the conditions on the spacetime geometry imposed by supersymmetry. In both cases, the spacetime admits a null, self-parallel, Killing vector field. We also apply our formalism to examine a class of $S U(4) \ltimes \mathbb{R}^{8}$ backgrounds which admit one and two pure spinors as Killing spinors and investigate the geometry of the spacetimes.
\end{abstract}




\section{Introduction}

In the last few years there has been much interest in the systematic understanding of supersymmetric solutions of ten- and eleven-dimensional supergravities. The maximal supersymmetric solutions of eleven-dimensional supergravity have been classified in [1, 2] by exploring the vanishing of the curvature of the supercovariant connection of the theory. The Killing spinor equations have also been solved for one Killing spinor and the geometry of the spacetime has been investigated in [3, 4]. This has been done by using the properties of spinor bilinears. The supersymmetric backgrounds of eleven-dimensional supergravity have also be examined using the holonomy of the supercovariant connection [5, 6, 7], see also [8] and [9]. In [10] it has been shown that that backgrounds with more than twenty four supersymmetries are locally homogeneous spaces.

Recently, a new method for solving the Killing spinor equations of supergravity theories has been proposed in [11 and applied to eleven-dimensional supergravity. This is based on a realization of spinors in terms of forms and the introduction of a basis in the space of spinors. Using this method, one can easily analyze the Killing spinor equations and determine the geometry of the associated spacetime. As a demonstration of the effectiveness of this method, the Killing spinor equations of eleven-dimensional supergravity have been solved for one, two, three and four spinors with stability subgroups $S U(5)$ and $S U(4)[1]$.

Some progress towards a systematic understanding of supersymmetric solutions of IIB supergravity [12, 13] has also been made. The maximal supersymmetric solutions of IIB supergravity been classified in [1, 2]. It has been found that they are locally isometric to Minkowski space, $A d S_{5} \times S^{5}[12$ and the maximal supersymmetric plane wave [14], and they are related by Penrose limits [15]. The holonomy of the the supercovariant connection of IIB supergravity is $S L(32, \mathbb{R})$ which reduces for backgrounds with $N$ spinors to a subgroup of $S L(32-N, \mathbb{R}) \ltimes \oplus_{N} \mathbb{R}^{32-N}$ [16]. In addition, the Killing spinor equations of IIB supergravity have been expressed as the parallel transport equations for the associated form bi-linears in [17. Other methods have also been used to solve the IIB Killing spinor equations like for example the 'algebraic spinor' technique which has been applied to construct supersymmetric flows [18].

In this paper, we use the method proposed in [1] to solve the Killing spinor equations of IIB supergravity for backgrounds that admit a $\operatorname{Spin}(7) \ltimes \mathbb{R}^{8}$ or an $S U(4) \ltimes \mathbb{R}^{8}$ invariant Killing spinor. As an application, we examine backgrounds that admit one and two $S U(4) \ltimes \mathbb{R}^{8}$ invariant pure Killing spinors. To apply the method, one has to take the following steps.

- Find a realization of spinors in terms of forms and construct a basis in the space of spinors.

- Find a canonical or normal form for the Killing spinors up to the gauge transformations of the Killing spinor equations of the supergravity theory.

- Substitute the canonical form of Killing spinors into the Killing spinor equations and use the basis in the space of spinors to turn the Killing spinor equations into a linear system for the fluxes, the geometry and the spacetime derivatives of functions that determine locally the Killing spinors. 
- Solve the linear system for the fluxes and find the conditions that arise on the geometry of the spacetime ${ }^{1}$.

A description of $\operatorname{Spin}(9,1)$ spinors in terms of forms is presented in appendix A. A suitable basis in the space of spinors for our analysis is also given. The manifest gauge invariance of the IIB supergravity depends on the formulation of the theory. If the supercovariant connection is written as in [12], then the gauge invariance is $\operatorname{Spin}(9,1) \times$ $U(1)$, where $U(1)$ is a local (duality) gauge group ${ }^{2}$. However, it is convenient not to fix the $U(1)$ part of the gauge symmetry because it may be used later to simplify computations for specific backgrounds, see [20]. Because of this, we use only the $\operatorname{Spin}(9,1)$ gauge group to bring the Killing spinor into a canonical form. We find that there are three cases to be considered which are distinguished by the stability subgroup of the Killing spinors in $\operatorname{Spin}(9,1)$. These are $\operatorname{Spin}(7) \ltimes \mathbb{R}^{8}, S U(4) \ltimes \mathbb{R}^{8}$ and $G_{2}$. The canonical forms of the Killing spinors written in terms of forms are

$$
\begin{aligned}
\epsilon & =(f+i g)\left(1+e_{1234}\right) \\
\epsilon & =\left(f-g_{2}+i g_{1}\right) 1+\left(f+g_{2}+i g_{1}\right) e_{1234} \\
\epsilon & =f\left(1+e_{1234}\right)+i \frac{g}{\sqrt{2}} \Gamma^{+}\left(e_{1}+e_{234}\right)
\end{aligned}
$$

respectively, where $f, g, g_{1}, g_{2}$ are real functions of the spacetime. The Killing spinors depend on more than one spacetime function.

We then substitute the $\operatorname{Spin}(7) \ltimes \mathbb{R}^{8}$ and $S U(4) \ltimes \mathbb{R}^{8}$ invariant spinors into the Killing spinor equations ${ }^{3}$. In both cases, the linear system that we derive, after expanding the Killing spinors in the spinor basis that we have constructed in appendix A, is rather involved. However all equations can be solved to express some of the fluxes in terms of the geometry and to find the conditions on the geometry imposed by supersymmetry. The expressions for the fluxes are simplified using the self-duality condition of the fiveform flux $F$.

The conditions on the geometry, which are expressed as relations between components of the Levi-Civita connection of the spacetime, can be directly analyzed to specify the geometry of the spacetime. For the $\operatorname{Spin}(7) \ltimes \mathbb{R}^{8}$ and $S U(4) \ltimes \mathbb{R}^{8}$ cases, it is also convenient to consider the spacetime form bi-linears associated with the Killing spinor. However unlike the eleven-dimensional supergravity case, the spinors of IIB supergravity are complex. Because of this acting on the Killing spinors with Pin and Spin invariant operations, one can construct new spinors that are defined on the spacetime. One such operator is $C *$, where $*$ is the standard complex conjugation and $C$ is a charge conjugation matrix (see appendix A). Therefore if $\epsilon$ is a Killing spinor, then $\tilde{\epsilon}=C\left(\epsilon^{*}\right)$ is a spinor defined on the spacetime but not always Killing. It turns out that for the geometric interpretation of the conditions on the Levi-Civita connection arising from the Killing spinor equations, it is necessary to construct the spacetime form bi-linears of the pairs $(\epsilon, \epsilon)$, $(\epsilon, \tilde{\epsilon})$ and $(\tilde{\epsilon}, \tilde{\epsilon})$. The spacetime of supersymmetric IIB backgrounds with $\operatorname{Spin}(7) \ltimes \mathbb{R}^{8}$ and $S U(4) \ltimes \mathbb{R}^{8}$ invariant Killing spinors admits a null, self-parallel, Killing vector field.

\footnotetext{
${ }^{1}$ The functions that the Killing spinors depend on may also be restricted.

${ }^{2}$ The field equations of IIB supergravity have an additional global $S L(2, \mathbb{R})$ symmetry 19,12

${ }^{3}$ The $G_{2}$ invariant case will be investigated elsewhere [21].
} 
In addition the associated spacetime admits a $\operatorname{Spin}(7) \ltimes \mathbb{R}^{8}$ and $S U(4) \ltimes \mathbb{R}^{8}$ structure, respectively. Examples of such spacetimes are Lorentzian extensions of one-parameter families of eight-dimensional manifolds with generic $\operatorname{Spin}(7)$ and $S U(4)$ structures.

A special class of IIB backgrounds with $S U(4) \ltimes \mathbb{R}^{8}$-invariant parallel spinors are those for which the Killing spinor is pure. Pure spinors have been considered before in relation to the Killing spinor equations of IIB supergravity in 22] in a somewhat different context. The definition of pure spinor can be given in different ways. One way ${ }^{4}$ is to assert that a spinor is pure iff the one-form bi-linear of the spinor vanishes. Using this definition, one can find that an $S U(4) \ltimes \mathbb{R}^{8}$-invariant spinor is pure if either

$$
\epsilon=h 1,
$$

i.e. $g_{1}=0$ and $f=-g_{2}=h / 2$, or

$$
\epsilon=k e_{1234}
$$

i.e. $g_{1}=0$ and $f=g_{2}=k / 2$. We shall analyze the conditions in both cases. In particular, we shall investigate the $\epsilon=h 1$ case in some detail. This is because it is rather straightforward to solve the Killing spinor equations and it has most of the features of the generic $S U(4) \ltimes \mathbb{R}^{8}$ invariant case. We shall also summarize the conditions required by supersymmetry for the $\epsilon=k e_{1234}$ Killing spinor. Then, we shall give the conditions required for both $h 1$ and $k e_{1234}$ to be Killing spinors and we shall investigate the geometry of the associated spacetime.

The $\operatorname{Spin}(7) \ltimes \mathbb{R}^{8}$ invariant Killing spinor is a special case of the $S U(4) \ltimes \mathbb{R}^{8}$ invariant one, as it can be seen by setting $g_{2}=0$ and $g_{1}=g$ in (1.1). Nevertheless it turns out that some of the conditions that arise on the geometry and some of the expressions for the fluxes are different. Because of this, we shall treat them as two distinct cases. We shall point out some of the differences in the geometry at the relevant sections.

This paper has been organized as follows: In section two, we use the $\operatorname{Spin}(9,1)$ gauge transformations of the Killing spinor equations of IIB supergravity to bring the Killing spinors to a canonical form. We also present the IIB Killing spinor equations and investigate some of their properties. In section three, we give the linear system that arises from the Killing spinor equations and summarize the conditions on the geometry of the spacetime that admits an $S U(4) \ltimes \mathbb{R}^{8}$ invariant Killing spinor. We also investigate these conditions using the spacetime-form bi-linears that are associated to the Killing spinor. In appendix B, we explain the derivation of the linear system and express the fluxes in terms of the geometry. In section four, we summarize the conditions on the geometry of the spacetime that admits a $\operatorname{Spin}(7) \ltimes \mathbb{R}^{8}$ invariant Killing spinor and investigate the geometry of the associated spacetime. In appendix $\mathrm{C}$, we express the fluxes in terms of the geometry for the $\operatorname{Spin}(7) \ltimes \mathbb{R}^{8}$ invariant Killing spinor. In section five, we present the conditions on the geometry for backgrounds with $h 1$ and $k e_{1234}$ pure Killing spinors. The fluxes for these cases are given in appendices D and E. In section six, we solve the Killing spinor equations for backgrounds which admit both $h 1$ and $k e_{1234}$ as Killing spinors and investigate the geometry of the associated spacetimes.

\footnotetext{
${ }^{4}$ Another way is to say that the pure spinor is annihilated by a maximal isotropic subspace of the spacetime, i.e. half of the gamma matrices along some directions annihilate the spinor.
} 


\section{Orbits of $\operatorname{Spin}(9,1)$ and Killing spinor equations}

\subsection{Orbits of $\operatorname{Spin}(9,1)$ in $\Delta_{16}^{+}$}

The group $\operatorname{Spin}(9,1)$ has one type of orbit with stability subgroup $\operatorname{Spin}(7) \ltimes \mathbb{R}^{8}$ in the Majorana-Weyl representation ${ }^{5} \Delta_{16}^{+}$. To see this, consider the spinor represented by

$$
1+e_{1234}
$$

The stability subgroup of this spinor in $\operatorname{Spin}(9,1)$ is $\operatorname{Spin}(7) \ltimes \mathbb{R}^{8}$. This can be easily seen by adapting a computation of [23] done for eleven-dimensional supergravity to this case, see also [24] and [25. To find the stability subgroup, we solve the infinitesimal equation

$$
\lambda_{A B} \Gamma^{A B}\left(1+e_{1234}\right)=0
$$

where $\lambda$ parameterizes the infinitesimal spinor transformations. This computation is most easily done in the pseudo-Hermitian basis (A.12). It is easy to see that the above condition implies that the parameters are restricted as

$$
\lambda_{\bar{\alpha} \bar{\beta}}=\frac{1}{2} \epsilon_{\bar{\alpha} \bar{\beta}}^{\gamma \delta} \lambda_{\gamma \delta}, \quad \lambda_{\alpha \bar{\beta}} g^{\alpha \bar{\beta}}=\lambda_{-+}=\lambda_{+\alpha}=\lambda_{+\bar{\alpha}}=0
$$

where $\epsilon_{\overline{1} \overline{2} \overline{3} \overline{4}}=1$. Observe that $\lambda_{-\alpha}$ and $\lambda_{-\bar{\alpha}}$ are complex conjugate to each other but otherwise unconstrained. It is known that the Lie algebra $\operatorname{spin}(7)$ in a Hermitian basis is spanned by traceless $(1,1)$-forms and $(2,0)$-forms in $\mathbb{C}^{4}$ which are related to their complex conjugates by a duality relation as in the first equation of (2.3), see e.g. [26]. Therefore the group that leaves invariant $1+e_{1234}$ has Lie algebra $\operatorname{spin}(7) \oplus \mathbb{R}^{8}$. So we shall take the stability subgroup ${ }^{6}$ to be $\operatorname{Spin}(7) \ltimes \mathbb{R}^{8}$. Note that the product is semi-direct because $\operatorname{Spin}(7)$ acts on $\mathbb{R}^{8}$ with a spin representation.

Having established this, we decompose $\Delta_{16}^{+}$under the stability subgroup $\operatorname{Spin}(7)$ as

$$
\Delta_{16}^{+}=\mathbb{R}<1+e_{1234}>+\Lambda^{1}\left(\mathbb{R}^{7}\right)+\Delta_{8}
$$

where the singlet $\mathbb{R}$ is generated by $1+e_{1234}, \Lambda^{1}\left(\mathbb{R}^{7}\right)$ is the vector representation of $\operatorname{Spin}(7)$ which is spanned by the Majorana spinors associated with two-forms in the directions $e_{1}, \ldots, e_{4}$ and $i\left(1-e_{1234}\right)$, and $\Delta_{8}$ is the spin representation of $\operatorname{Spin}(7)$ which is spanned by the rest of Majorana spinors which are of the type $\Gamma^{+} \eta, \eta$ is a spinor generated by the odd forms in the directions $e_{1}, \ldots, e_{4}$. Therefore the most general spinor in $\Delta_{16}^{+}$can be written as

$$
\eta=a\left(1+e_{1234}\right)+\theta_{1}+\theta_{2}
$$

where $\theta_{1} \in \Lambda^{1}\left(\mathbb{R}^{7}\right)$ and $\theta_{2} \in \Delta_{8}$. First we assume that $a \neq 0$. In this case, there are two cases to consider depending on whether $\theta_{2}$ vanishes or not. If $\theta_{2}=0$, since $\operatorname{Spin}(7)$ acts with the vector representation on $\Lambda^{1}\left(\mathbb{R}^{7}\right)$, it is always possible to choose $\theta_{1}=i b\left(1-e_{1234}\right)$. The most general spinor in this case then is

$$
\eta=a\left(1+e_{1234}\right)+i b\left(1-e_{1234}\right) .
$$

\footnotetext{
${ }^{5}$ Our spinor conventions as well as the realization of spinors in terms of forms is explained in detail in appendix $\mathrm{A}$.

${ }^{6}$ There may be subtleties with discrete groups, see [27, 28].
} 
However, it is easy to see that this spinor is in the same orbit as $1+e_{1234}$, e.g. observe that

$$
\eta=h e^{\psi \Gamma_{16}}\left(1+e_{1234}\right)
$$

where $h^{2}=a^{2}+b^{2}$ and $\tan \psi=b / a$. Next suppose that $\theta_{2}$ does not vanish. If $\theta_{2} \neq 0$, there is always a $\operatorname{Spin}(7)$ transformation such that $\theta_{2}=c \Gamma^{+}\left(e_{1}+e_{234}\right)$. This is because $\operatorname{Spin}(7)$ acts transitively on the $S^{7}$ in $\Delta_{8}$ and the stability subgroup is $G_{2}, \operatorname{Spin}(7) / G_{2}=S^{7}$. In addition $G_{2}$ acts transitively on the $S^{6}$ in $\Lambda^{1}\left(\mathbb{R}^{7}\right)$ with stability subgroup $S U(3)$, see e.g. [26]. So it can always be arranged such that $\theta_{1}=i b\left(1-e_{1234}\right)$. Therefore the most general spinor in this case is

$$
\eta=a\left(1+e_{1234}\right)+i b\left(1-e_{1234}\right)+c \Gamma^{+}\left(e_{1}+e_{234}\right)
$$

However observe that this spinor is in the same orbit as $1+e_{1234}$. Indeed

$$
\eta=e^{\frac{b}{2 c} \Gamma^{-} \Gamma^{6}} e^{\frac{c}{a} \Gamma^{+} \Gamma^{1}} a\left(1+e_{1234}\right) .
$$

So, we conclude that if $a \neq 0$, then there is one orbit represented by $a\left(1+e_{1234}\right)$. It remains to investigate the case where $a=0$. In this case, it is straightforward to see that the orbit can always be represented by $c \Gamma^{+}\left(e_{1}+e_{234}\right)$. In turn, this spinor is in the same orbit of $\operatorname{Spin}(9,1)$ as $\frac{c}{\sqrt{2}}\left(1+e_{1234}\right)$ as can seen by acting on the latter with the element $\Gamma_{5} \Gamma_{1}$ of $\operatorname{Spin}(9,1)$. As a consequence, the stability subgroup of $c \Gamma^{+}\left(e_{1}+e_{234}\right)$ is again $\operatorname{Spin}(7) \ltimes \mathbb{R}^{8}$. Therefore we conclude that there is only one type of orbit of $\operatorname{Spin}(9,1)$ in $\Delta_{16}^{+}$which can be represented with $a\left(1+e_{1234}\right)$.

In IIB supergravity, the Killing spinors are (complex) Weyl and so they take values in two copies of the same Majorana-Weyl representation $\Delta_{16}^{+}$. To find the most general Killing spinor that can arise in the theory, we assume that the Killing spinor in the first copy is represented by $a\left(1+e_{1234}\right)$ and decompose the second Majorana-Weyl representation under the stability subgroup $\operatorname{Spin}(7) \ltimes \mathbb{R}^{8}$ of $\eta_{1}$. As we have mentioned, $\Delta_{16}^{+}$under $\operatorname{Spin}(7)$ decomposes as (2.4). Using the same arguments as those below (2.4), we can choose the two Majorana Weyl spinors of IIB supergravity to take the form

$$
\begin{aligned}
& \eta_{1}=a\left(1+e_{1234}\right) \\
& \eta_{2}=b_{1}\left(1+e_{1234}\right)+i b_{2}\left(1-e_{1234}\right)+b_{3} \Gamma^{+}\left(e_{1}+e_{234}\right) .
\end{aligned}
$$

So far we have been concerned with the orbits of $\operatorname{Spin}(7)$. At this point we have to distinguish between two different cases. First suppose that $b_{3} \neq 0$. In this case, we can further consider the action of $\mathbb{R}^{8}$ on the (2.10) spinors. In particular observe that

$$
\eta_{2}=e^{-\frac{b_{1}}{2 b_{3}} \Gamma^{-} \Gamma^{6}+\frac{b_{2}}{2 b_{3}} \Gamma^{-} \Gamma^{1}} b_{3} \Gamma^{+}\left(e_{1}+e_{234}\right)
$$

Therefore, we have shown that in this case the two Majorana-Weyl spinors of IIB supergravity can be represented by $\eta_{1}=a\left(1+e_{1234}\right)$ and $\eta_{2}=b \Gamma^{+}\left(e_{1}+e_{234}\right)$. The canonical form of the spinors is

$$
\begin{aligned}
& \eta_{1}=f\left(1+e_{1234}\right) \\
& \eta_{2}=g \Gamma^{+}\left(e_{1}+e_{234}\right)
\end{aligned}
$$


where $f, g$ are real spacetime functions. In particular, the Killing spinor in (2.17) and (2.18) below is $\epsilon=\eta_{1}+i \eta_{2}$. The stability subgroup of the Killing spinor is $G_{2}$.

Next suppose that $b_{3}=0$. In this case, we have

$$
\begin{aligned}
& \eta_{1}=f\left(1+e_{1234}\right), \\
& \eta_{2}=g_{1}\left(1+e_{1234}\right)+i g_{2}\left(1-e_{1234}\right)
\end{aligned}
$$

where $f, g_{1}$ and $g_{2}$ are real spacetime functions. The Killing spinor in (2.17) and (2.18) below is $\epsilon=\eta_{1}+i \eta_{2}$. The stability subgroup of the Killing spinor is $S U(4) \ltimes \mathbb{R}^{8}$.

One can also take $b_{3}=b_{2}=0$, in this case we have

$$
\begin{aligned}
& \eta_{1}=f\left(1+e_{1234}\right) \\
& \eta_{2}=g\left(1+e_{1234}\right),
\end{aligned}
$$

where $f, g$ are real spacetime functions. Similarly, the Killing spinor in (2.17) and (2.18) below is $\epsilon=\eta_{1}+i \eta_{2}$ and has stability subgroup $\operatorname{Spin}(7) \ltimes \mathbb{R}^{8}$.

To summarize, there are three types of orbits of $\operatorname{Spin}(9,1)$ in the Weyl spinor representation with stability subgroups $\operatorname{Spin}(7) \ltimes \mathbb{R}^{8}, S U(4) \ltimes \mathbb{R}^{8}$ and $G_{2}$. The representatives of these orbits are given in (1.1). Unlike the M-theory case, the Killing spinor depend on more than one spacetime function. It is straightforward to extend the analysis in this section to backgrounds which have more than one supersymmetry.

\subsection{Killing spinor equations}

The bosonic fields of IIB supergravity are the spacetime metric $g$, two real scalars, the axion $\sigma$ and the dilaton $\phi$, two three-form field strengths $G_{1}$ and $G_{2}$, and a self-dual fiveform field strength $F$. The Killing spinor equations of IIB supergravity are the parallel transport equations of the supercovariant derivative $\mathcal{D}[12$

$\mathcal{D}_{M} \epsilon=\tilde{\nabla}_{M} \epsilon+\frac{i}{480} \Gamma^{N_{1} \ldots N_{5}} \Gamma_{M} \epsilon F_{N_{1} \ldots N_{5}}-\frac{1}{96}\left(\Gamma_{M}^{N_{1} N_{2} N_{3}} G_{N_{1} N_{2} N_{3}}-9 \Gamma^{N_{1} N_{2}} G_{M N_{1} N_{2}}\right)(C \epsilon)^{*}=0$,

and the algebraic condition

$$
P_{M} \Gamma^{M}(C \epsilon)^{*}+\frac{1}{24} G_{N_{1} N_{2} N_{3}} \Gamma^{N_{1} N_{2} N_{3}} \epsilon=0
$$

where

$$
\tilde{\nabla}_{M}=D_{M}+\frac{1}{4} \Omega_{M, A B} \Gamma^{A B}, \quad D_{M}=\partial_{M}-\frac{i}{2} Q_{M}
$$

is the spin connection, $\nabla_{M}=\partial_{M}+\frac{1}{4} \Omega_{M, A B} \Gamma^{A B}$, twisted with $U(1)$ connection $Q_{M}$, $Q_{M}^{*}=Q_{M}, \epsilon$ is a (complex) Weyl spinor, $\Gamma^{0 \ldots 9} \epsilon=-\epsilon$, and $C$ is a charge conjugation matrix $^{7}$. (For our spinor conventions see appendix A.) Killing spinor equations are the vanishing conditions of the supersymmetry transformations of the gravitino, and the supersymmetric partners of the dilaton and axion restricted to the bosonic sector of IIB 29].

${ }^{7}$ In the basis of gamma matrices chosen in [12],C=1, and so it has been neglected, see however 
supergravity, respectively. The precise dependence of the complex field strengths $G$ and $P$ on the scalars and $G_{1}, G_{2}$ field strengths is described ${ }^{8}$ in [12] and we shall not repeat the formulae here. For a superspace formulation of IIB supergravity see [13.

It is convenient to choose the orientation of spacetime as $\epsilon_{01 \ldots 9}=1$. In this case, the self-duality condition on $F$ is $F_{M_{1} \ldots M_{5}}=-\frac{1}{5 !} \epsilon_{M_{1} \ldots M_{5}}{ }^{N_{1} \ldots N_{5}} F_{N_{1} \ldots N_{5}}$. Because $\epsilon$ is chiral, the Killing spinor equations can be rewritten as

$$
\tilde{\nabla}_{M} \epsilon+\frac{i}{48} \Gamma^{N_{1} \ldots N_{4}} \epsilon F_{N_{1} \ldots N_{4} M}-\frac{1}{96}\left(\Gamma_{M}{ }^{N_{1} N_{2} N_{3}} G_{N_{1} N_{2} N_{3}}-9 \Gamma^{N_{1} N_{2}} G_{M N_{1} N_{2}}\right)(C \epsilon)^{*}=0
$$

and

$$
P_{M} \Gamma^{M}(C \epsilon)^{*}+\frac{1}{24} G_{N_{1} N_{2} N_{3}} \Gamma^{N_{1} N_{2} N_{3}} \epsilon=0 .
$$

Observe that the above Killing spinor equations are at most fourth order in the gamma matrices.

It is worth exploring some general properties of the above Killing spinor equations. Acting with the anti-linear map $C *$, the Killing spinor equations (2.17) and (2.18) become

$$
\begin{gathered}
\nabla_{M}(C \epsilon)^{*}+\frac{i}{2} Q_{M}(C \epsilon)^{*}-\frac{i}{48} \Gamma^{N_{1} \ldots N_{4}}(C \epsilon)^{*} F_{N_{1} \ldots N_{4} M} \\
-\frac{1}{96}\left(\Gamma_{M}^{N_{1} N_{2} N_{3}} G_{N_{1} N_{2} N_{3}}^{*}-9 \Gamma^{N_{1} N_{2}} G_{M N_{1} N_{2}}^{*}\right) \epsilon=0
\end{gathered}
$$

where $\nabla$ is the spin connection and $Q_{M}^{*}=Q_{M}$, and

$$
P_{M}^{*} \Gamma^{M} \epsilon+\frac{1}{24} G_{N_{1} N_{2} N_{3}}^{*} \Gamma^{N_{1} N_{2} N_{3}}(C \epsilon)^{*}=0
$$

respectively. Comparing (2.17), (2.18) and (2.19), (2.20), it is clear that if $\epsilon$ is a parallel spinor, then $(C \epsilon)^{*}$ is also a parallel spinor provided that the fluxes $P, G$ are real and $F=0$. In such a case, the background will have at least two parallel spinors.

Next suppose that $\epsilon$ is a Majorana-Weyl spinor. The Majorana condition implies that $(C \epsilon)^{*}=\epsilon$. This can be used to rewrite the Killing spinor equations (2.17) and (2.18), and (2.19) and (2.20), in terms of $\epsilon$ only. Taking the difference of (2.17) and (2.19), we find

$$
\begin{array}{r}
-i Q_{M} \epsilon+\frac{i}{24} \Gamma^{N_{1} \ldots N_{4}} \epsilon F_{N_{1} \ldots N_{4} M}-\frac{1}{96}\left[\Gamma_{M}^{N_{1} N_{2} N_{3}}\left(G-G^{*}\right)_{N_{1} N_{2} N_{3}}\right. \\
\left.-9 \Gamma^{N_{1} N_{2}}\left(G-G^{*}\right)_{M N_{1} N_{2}}\right] \epsilon=0 .
\end{array}
$$

Similarly, taking the difference of (2.18) and (2.20), we find

$$
\left(P-P^{*}\right)_{M} \Gamma^{M} \epsilon+\frac{1}{24}\left(G-G^{*}\right)_{N_{1} N_{2} N_{3}} \Gamma^{N_{1} N_{2} N_{3}} \epsilon=0 .
$$

It appears that the Majorana condition on the spinor imposes some reality restrictions on the complex field strengths $P$ and $G$.

\footnotetext{
${ }^{8}$ We use a mostly plus convention for the metric. To relate this to the conventions of [12, one takes $\Gamma^{A} \rightarrow i \Gamma^{A}$ and every time a index is lowered there is also an additional minus sign.
} 


\section{$3 \quad S U(4) \ltimes \mathbb{R}^{8}$-invariant Killing spinors}

\subsection{The conditions}

We have shown that the canonical form of the most general IIB $S U(4) \ltimes \mathbb{R}^{8}$ invariant Killing spinor is $\epsilon=\left(f-g_{2}+i g_{1}\right) 1+\left(f+g_{2}+i g_{1}\right) e_{1234}$, where $f, g_{1}, g_{2}$ are real functions. Using the property that $\Gamma^{-} \epsilon=0$, one can show that

$$
\epsilon^{w \Gamma^{-+}} \epsilon=e^{w} \epsilon
$$

for any spacetime function $w$. This gauge freedom can be used to normalize the overall scale of $\epsilon$. We shall use this freedom in the description of geometry of supersymmetric backgrounds.

To derive the linear system which does not involve gamma matrices from the Killing spinor equations, we substitute the above spinor $\epsilon$ into the Killing spinor equations. Then we decompose the vector $S O(9,1)$ representation under $S U(4)$. This is equivalent to decomposing the frame indices as $A=(+,-, \alpha, \bar{\alpha})$. Consequently, the fluxes and geometry decompose into $S U(4)$ representations, i.e. $P_{A}$ decomposes as $P_{+}, P_{-}, P_{\alpha}$ and $P_{\bar{\alpha}}$ and similarly for the other fluxes and geometry ${ }^{9}$. We also decompose the Killing spinor equations under $S U(4)$ representations using the decomposition of the fluxes and geometry that we have mentioned and the decomposition the gamma matrices as $\Gamma^{A}=$ $\left(\Gamma^{+}, \Gamma^{-}, \Gamma^{\alpha}, \Gamma^{\bar{\alpha}}\right)$. Then we use the properties $\Gamma^{\alpha} 1=\Gamma^{-} 1=0$ and $\Gamma^{\bar{\alpha}} e_{1234}=\Gamma^{-} e_{1234}=0$, which we have explained in appendix A, to rewrite the Killing spinor equations in the (A.13) basis. Setting every component of the Killing spinor equations in this basis to zero, we derive a linear system for the fluxes, the geometry, as represented by the LeviCivita connection of spacetime, and the first derivatives of the functions $f, g_{1}$ and $g_{2}$. Here, we shall present the linear system that arises from the Killing spinor equations. This system is solved in appendix B.

First, we substitute this spinor into the (algebraic) Killing spinor equation (2.18), expand the resulting expression in the basis (A.13) and set every component in this basis to zero. We find that (2.18) implies the conditions

$$
\begin{gathered}
\left(f+g_{2}-i g_{1}\right) P_{\bar{\alpha}}+\frac{1}{4}\left(f-g_{2}+i g_{1}\right) G_{-+\bar{\alpha}}+\frac{1}{4}\left(f-g_{2}+i g_{1}\right) G_{\bar{\alpha} \beta}{ }^{\beta} \\
+\frac{1}{12}\left(f+g_{2}+i g_{1}\right) \epsilon_{\bar{\alpha}}^{\beta_{1} \beta_{2} \beta_{3}} G_{\beta_{1} \beta_{2} \beta_{3}}=0 \\
\left(f-g_{2}-i g_{1}\right) P_{\alpha}+\frac{1}{4}\left(f+g_{2}+i g_{1}\right) G_{-+\alpha}-\frac{1}{4}\left(f+g_{2}+i g_{1}\right) G_{\alpha \beta}{ }^{\beta} \\
+\frac{1}{12}\left(f-g_{2}+i g_{1}\right) \epsilon_{\alpha}{ }^{\overline{\beta_{1}} \bar{\beta}_{2} \bar{\beta}_{3}} G_{\overline{\beta_{1}} \overline{\beta_{2}} \overline{\beta_{3}}}=0 \\
\left(f+g_{2}-i g_{1}\right) P_{+}+\frac{1}{4}\left(f-g_{2}+i g_{1}\right) G_{+\alpha}{ }^{\alpha}=0
\end{gathered}
$$

\footnotetext{
${ }^{9}$ If the fluxes are complex, like $P$ and $G$, then their various components do not satisfy the 'naive' complex conjugate relations, i.e. $\left(P_{\alpha}\right)^{*} \neq P_{\bar{\alpha}}$ and similarly for $G$.
} 


$$
\left(f-g_{2}-i g_{1}\right) P_{+}-\frac{1}{4}\left(f+g_{2}+i g_{1}\right) G_{+\alpha}{ }^{\alpha}=0
$$

and

$$
\left(f-g_{2}+i g_{1}\right) G_{+\bar{\alpha} \bar{\beta}}-\frac{1}{2}\left(f+g_{2}+i g_{1}\right) \epsilon_{\bar{\alpha} \bar{\beta}}{ }^{\gamma \delta} G_{+\gamma \delta}=0 .
$$

It is clear that this is a linear system for the fluxes of IIB supergravity and does not involve gamma matrices.

Next we turn into the Killing spinor equation associated with the supercovariant derivative (2.17) . In particular the conditions along the $\alpha$-frame derivative of the supercovariant connection are

$$
\begin{aligned}
& {\left[D_{\alpha}+\frac{1}{2} \Omega_{\alpha, \beta} \beta+\frac{1}{2} \Omega_{\alpha,-+}+\frac{i}{4} F_{\alpha \beta}{ }^{\beta} \gamma^{\gamma}+\frac{i}{2} F_{\alpha-+\beta}{ }^{\beta}\right]\left(f-g_{2}+i g_{1}\right)} \\
& +\left(f+g_{2}-i g_{1}\right)\left[\frac{1}{4} G_{\alpha \beta}{ }^{\beta}+\frac{1}{4} G_{-+\alpha}\right]=0, \\
& \left(f-g_{2}+i g_{1}\right)\left[\Omega_{\alpha, \bar{\beta}_{1} \bar{\beta}_{2}}+i F_{\alpha \bar{\beta}_{1} \bar{\beta}_{2 \gamma}}{ }^{\gamma}+i F_{\alpha-+\bar{\beta}_{1} \bar{\beta}_{2}}\right] \\
& +\left(f+g_{2}-i g_{1}\right)\left[\frac{1}{2} G_{\alpha \bar{\beta}_{1} \bar{\beta}_{2}}-\frac{1}{4} g_{\alpha\left[\bar{\beta}_{1}\right.} G_{\left.\bar{\beta}_{2}\right] \gamma}{ }^{\gamma}-\frac{1}{4} g_{\alpha\left[\bar{\beta}_{1}\right.} G_{\left.\bar{\beta}_{2}\right]-+}\right] \\
& -\left(f+g_{2}+i g_{1}\right)\left[\frac{1}{2} \Omega_{\alpha, \gamma_{1} \gamma_{2}}-\frac{i}{2} F_{\alpha \gamma_{1} \gamma_{2} \delta} \delta+\frac{i}{2} F_{\alpha-+\gamma_{1} \gamma_{2}}\right] \epsilon^{\gamma_{1} \gamma_{2}} \bar{\beta}_{1} \bar{\beta}_{2} \\
& -\frac{1}{8}\left(f-g_{2}-i g_{1}\right) G_{\alpha \gamma_{1} \gamma_{2}} \epsilon^{\gamma_{1} \gamma_{2}} \bar{\beta}_{1} \bar{\beta}_{2}=0 \text {, } \\
& {\left[D_{\alpha}-\frac{1}{2} \Omega_{\alpha, \beta}{ }^{\beta}+\frac{1}{2} \Omega_{\alpha,-+}+\frac{i}{4} F_{\alpha \beta}{ }^{\beta} \gamma^{\gamma}-\frac{i}{2} F_{\alpha-+\gamma}{ }^{\gamma}\right]\left(f+g_{2}+i g_{1}\right)} \\
& +\left[-\frac{1}{8} G_{\alpha \gamma}^{\gamma}+\frac{1}{8} G_{-+\alpha}\right]\left(f-g_{2}-i g_{1}\right)
\end{aligned}
$$

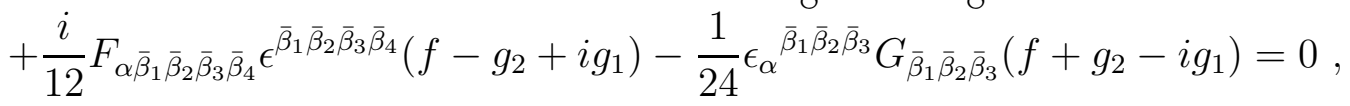

$$
\begin{aligned}
& {\left[\frac{1}{2} \Omega_{\alpha,+\bar{\beta}}+\frac{i}{2} F_{\alpha+\bar{\beta} \gamma}{ }^{\gamma}\right]\left(f-g_{2}+i g_{1}\right)+\left[\frac{1}{16} g_{\alpha \bar{\beta}} G_{+\gamma}^{\gamma}-\frac{1}{4} G_{+\alpha \bar{\beta}}\right]\left(f+g_{2}-i g_{1}\right)} \\
& -\frac{i}{6} F_{\alpha+\beta_{1} \beta_{2} \beta_{3}} \epsilon^{\beta_{1} \beta_{2} \beta_{3}}{ }_{\bar{\beta}}\left(f+g_{2}+i g_{1}\right)=0 \\
& \frac{i}{12} F_{\alpha+\bar{\beta}_{1} \bar{\beta}_{2} \bar{\beta}_{3}}\left(f-g_{2}+i g_{1}\right)+\frac{1}{32} g_{\alpha\left[\bar{\beta}_{1}\right.} G_{\left.\bar{\beta}_{2} \bar{\beta}_{3}\right]+}\left(f+g_{2}-i g_{1}\right)+\frac{1}{12}\left(f+g_{2}+i g_{1}\right) \\
& {\left[\frac{1}{2} \Omega_{\alpha,+\gamma}-\frac{i}{2} F_{\alpha+\gamma \delta}{ }^{\delta}\right] \epsilon_{\bar{\beta}_{1} \bar{\beta}_{2} \bar{\beta}_{3}}-\frac{1}{96}\left(f-g_{2}-i g_{1}\right) G_{+\alpha \gamma} \epsilon^{\gamma} \bar{\beta}_{1} \bar{\beta}_{2} \bar{\beta}_{3}=0 .}
\end{aligned}
$$

The conditions along the $\bar{\alpha}$-frame derivative of the supercovariant connection are

$$
\begin{aligned}
& {\left[D_{\bar{\alpha}}+\frac{1}{2} \Omega_{\bar{\alpha}, \beta}^{\beta}+\frac{1}{2} \Omega_{\bar{\alpha},-+}+\frac{i}{4} F_{\bar{\alpha} \beta}{ }^{\beta} \gamma^{\gamma}+\frac{i}{2} F_{\bar{\alpha}-+\beta}{ }^{\beta}\right]\left(f-g_{2}+i g_{1}\right)} \\
& +\frac{1}{8}\left[G_{\bar{\alpha} \beta}{ }^{\beta}+G_{\bar{\alpha}-+}\right]\left(f+g_{2}-i g_{1}\right)+\frac{i}{12}\left(f+g_{2}+i g_{1}\right) F_{\bar{\alpha} \gamma_{1} \gamma_{2} \gamma_{2} \gamma_{4}} \epsilon^{\gamma_{1} \gamma_{2} \gamma_{3} \gamma_{4}}
\end{aligned}
$$




$$
\begin{aligned}
& -\frac{1}{24}\left(f-g_{2}-i g_{1}\right) \epsilon_{\bar{\alpha}}^{\gamma_{1} \gamma_{2} \gamma_{3}} G_{\gamma_{1} \gamma_{2} \gamma_{3}}=0 \\
& {\left[\Omega_{\bar{\alpha}, \bar{\beta}_{1} \bar{\beta}_{2}}+i F_{\bar{\alpha} \bar{\beta}_{1} \bar{\beta}_{2} \gamma}^{\gamma}+i F_{\bar{\alpha}-+\bar{\beta}_{1} \bar{\beta}_{2}}\right]\left(f-g_{2}+i g_{1}\right)+\frac{1}{4} G_{\bar{\alpha} \bar{\beta}_{1} \bar{\beta}_{2}}\left(f+g_{2}-i g_{1}\right)} \\
& -\left(f+g_{2}+i g_{1}\right)\left[\frac{1}{2} \Omega_{\bar{\alpha}, \gamma_{1} \gamma_{2}}-\frac{i}{2} F_{\bar{\alpha} \gamma_{1} \gamma_{2} \delta} \delta+\frac{i}{2} F_{\bar{\alpha}-+\gamma_{1} \gamma_{2}}\right] \epsilon^{\gamma_{1} \gamma_{2}} \bar{\beta}_{1} \bar{\beta}_{2} \\
& -\left(f-g_{2}-i g_{1}\right)\left[\frac{1}{8} g_{\bar{\alpha} \gamma_{1}} G_{\gamma_{2} \delta} \delta+\frac{1}{4} G_{\bar{\alpha} \gamma_{1} \gamma_{2}}-\frac{1}{8} g_{\bar{\alpha} \gamma_{1}} G_{\gamma_{2}-+}\right] \epsilon^{\gamma_{1} \gamma_{2}}{ }_{\bar{\beta}_{1} \bar{\beta}_{2}}=0 \text {, } \\
& {\left[D_{\bar{\alpha}}-\frac{1}{2} \Omega_{\bar{\alpha}, \gamma} \gamma+\frac{1}{2} \Omega_{\bar{\alpha},-+}+\frac{i}{4} F_{\bar{\alpha} \gamma} \gamma_{\delta}^{\delta}-\frac{i}{2} F_{\bar{\alpha}-+\gamma}{ }^{\gamma}\right]\left(f+g_{2}+i g_{1}\right)} \\
& +\left[-\frac{1}{4} G_{\bar{\alpha} \gamma}{ }^{\gamma}+\frac{1}{4} G_{\bar{\alpha}-+}\right]\left(f-g_{2}-i g_{1}\right)=0, \\
& {\left[\frac{1}{2} \Omega_{\bar{\alpha},+\bar{\beta}}+\frac{i}{2} F_{\bar{\alpha}+\bar{\beta} \gamma}^{\gamma}\right]\left(f-g_{2}+i g_{1}\right)-\frac{1}{8} G_{+\bar{\alpha} \bar{\beta}}\left(f+g_{2}-i g_{1}\right)} \\
& -\frac{i}{6}\left(f+g_{2}+i g_{1}\right) F_{\bar{\alpha}+\gamma_{1} \gamma_{2} \gamma_{3}} \epsilon^{\gamma_{1} \gamma_{2} \gamma_{3}}{ }_{\bar{\beta}}-\frac{1}{16}\left(f-g_{2}-i g_{1}\right) G_{\gamma_{1} \gamma_{2}+} \epsilon_{\bar{\alpha} \bar{\beta}}^{\gamma_{1} \gamma_{2}}=0 \text {, } \\
& i F_{\bar{\alpha}+\bar{\beta}_{1} \bar{\beta}_{2} \bar{\beta}_{3}}\left(f-g_{2}+i g_{1}\right)+\left(f+g_{2}+i g_{1}\right)\left[\frac{1}{2} \Omega_{\bar{\alpha},+\gamma}-\frac{i}{2} F_{\bar{\alpha}+\gamma \delta} \delta\right] \epsilon^{\gamma} \bar{\beta}_{1} \bar{\beta}_{2} \bar{\beta}_{3} \\
& +\left(f-g_{2}-i g_{1}\right)\left[-\frac{1}{16} g_{\bar{\alpha} \gamma} G_{+\delta}{ }^{\delta}+\frac{1}{4} G_{\bar{\alpha}+\gamma}\right] \epsilon^{\gamma} \bar{\beta}_{1} \bar{\beta}_{2} \bar{\beta}_{3}=0 \text {. }
\end{aligned}
$$

The conditions along the --frame derivative of the supercovariant connection are

$$
\begin{aligned}
& {\left[D_{-}+\frac{1}{2} \Omega_{-, \gamma}^{\gamma}+\frac{1}{2} \Omega_{-,-+}+\frac{i}{4} F_{-\gamma}^{\gamma} \delta^{\delta}\right]\left(f-g_{2}+i g_{1}\right)+\frac{1}{4} G_{-\gamma}^{\gamma}\left(f+g_{2}-i g_{1}\right)} \\
& +\frac{i}{12}\left(f+g_{2}+i g_{1}\right) F_{-\gamma_{1} \gamma_{2} \gamma_{3} \gamma_{4}} \epsilon^{\gamma_{1} \gamma_{2} \gamma_{3} \gamma_{4}}=0 \\
& {\left[\Omega_{-, \bar{\beta}_{1} \bar{\beta}_{2}}+i F_{-\bar{\beta}_{1} \bar{\beta}_{2} \gamma}{ }^{\gamma}\right]\left(f-g_{2}+i g_{1}\right)+\frac{1}{2} G_{-\bar{\beta}_{1} \bar{\beta}_{2}}\left(f+g_{2}-i g_{1}\right)} \\
& -\left(f+g_{2}+i g_{1}\right)\left[\frac{1}{2} \Omega_{-, \gamma_{1} \gamma_{2}}-\frac{i}{2} F_{-\gamma_{1} \gamma_{2} \delta} \delta\right] \epsilon^{\gamma_{1} \gamma_{2}} \bar{\beta}_{1} \bar{\beta}_{2} \\
& -\frac{1}{4}\left(f-g_{2}-i g_{1}\right) G_{-\gamma_{1} \gamma_{2}} \epsilon^{\gamma_{1} \gamma_{2}} \bar{\beta}_{1} \bar{\beta}_{2}=0 \text {, } \\
& {\left[D_{-}-\frac{1}{2} \Omega_{-, \gamma}^{\gamma}+\frac{1}{2} \Omega_{-,-+}+\frac{i}{4} F_{-\gamma}^{\gamma} \delta^{\delta}\right]\left(f+g_{2}+i g_{1}\right)} \\
& -\frac{1}{4}\left(f-g_{2}-i g_{1}\right) G_{-\gamma}^{\gamma}+\frac{i}{12}\left(f-g_{2}+i g_{1}\right) F_{-\bar{\beta}_{1} \bar{\beta}_{2} \bar{\beta}_{3} \bar{\beta}_{4}} \epsilon^{\bar{\beta}_{1} \bar{\beta}_{2} \bar{\beta}_{3} \bar{\beta}_{4}}=0 \text {, }
\end{aligned}
$$

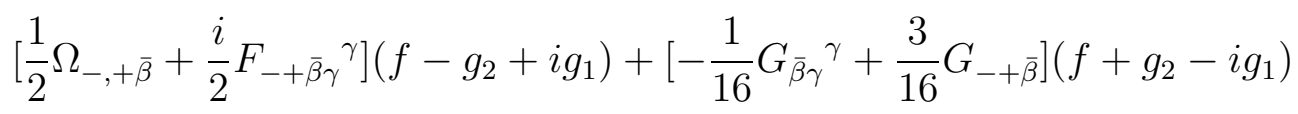




$$
\begin{gathered}
-\frac{i}{6}\left(f+g_{2}+i g_{1}\right) F_{-+\gamma_{1} \gamma_{2} \gamma_{3}} \epsilon^{\gamma_{1} \gamma_{2} \gamma_{3}}+\frac{1}{48}\left(f-g_{2}-i g_{1}\right) G_{\gamma_{1} \gamma_{2} \gamma_{3}} \epsilon^{\gamma_{1} \gamma_{2} \gamma_{3}}{ }_{\bar{\beta}}=0 \\
i F_{-+\bar{\beta}_{1} \bar{\beta}_{2} \bar{\beta}_{3}}\left(f-g_{2}+i g_{1}\right)-\frac{1}{8} G_{\bar{\beta}_{1} \bar{\beta}_{2} \bar{\beta}_{3}}\left(f+g_{2}-i g_{1}\right) \\
\left.+\left[\frac{1}{2} \Omega_{-,+\gamma}-\frac{i}{2} F_{-+\gamma \delta}\right]^{\delta}\right] \epsilon^{\gamma} \bar{\beta}_{1} \bar{\beta}_{2} \bar{\beta}_{3}\left(f+g_{2}+i g_{1}\right) \\
+\left[\frac{1}{16} G_{\gamma \delta} \delta+\frac{3}{16} G_{-+\gamma}\right]\left(f-g_{2}-i g_{1}\right) \epsilon^{\gamma} \bar{\beta}_{1} \bar{\beta}_{2} \bar{\beta}_{3}=0
\end{gathered}
$$

The conditions along the +-frame derivative of the supercovariant connection are

$$
\begin{aligned}
& {\left[D_{+}+\frac{1}{2} \Omega_{+, \gamma}^{\gamma}+\frac{1}{2} \Omega_{+,-+}+\frac{i}{4} F_{+\gamma}^{\gamma} \delta^{\delta}\right]\left(f-g_{2}+i g_{1}\right)} \\
& +\frac{1}{8} G_{+\gamma}^{\gamma}\left(f+g_{2}-i g_{1}\right)+\frac{i}{12}\left(f+g_{2}+i g_{1}\right) F_{+\gamma_{1} \gamma_{2} \gamma_{3} \gamma_{4}} \epsilon^{\gamma_{1} \gamma_{2} \gamma_{3} \gamma_{4}}=0 \\
& {\left[\Omega_{+, \bar{\beta}_{1} \bar{\beta}_{2}}+i F_{+\bar{\beta}_{1} \bar{\beta}_{2} \gamma}{ }^{\gamma}\right]\left(f-g_{2}+i g_{1}\right)+\frac{1}{4}\left(f+g_{2}-i g_{1}\right) G_{+\bar{\beta}_{1} \bar{\beta}_{2}}} \\
& -\left[\frac{1}{2} \Omega_{+, \gamma_{1} \gamma_{2}}-\frac{i}{2} F_{+\gamma_{1} \gamma_{2} \delta} \delta\right]\left(f+g_{2}+i g_{1}\right) \epsilon^{\gamma_{1} \gamma_{2}} \bar{\beta}_{1} \bar{\beta}_{2} \\
& -\frac{1}{8}\left(f-g_{2}-i g_{1}\right) G_{+\gamma_{1} \gamma_{2}} \epsilon^{\gamma_{1} \gamma_{2}} \bar{\beta}_{1} \bar{\beta}_{2}=0 \text {, } \\
& {\left[D_{+}-\frac{1}{2} \Omega_{+, \gamma}^{\gamma}+\frac{1}{2} \Omega_{+,-+}+\frac{i}{4} F_{+\gamma}^{\gamma} \delta^{\delta}\right]\left(f+g_{2}+i g_{1}\right)}
\end{aligned}
$$

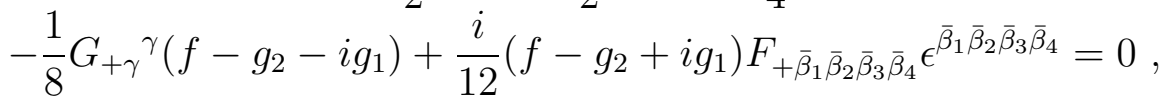

and

$$
\Omega_{+,+\alpha}=\Omega_{+,+\bar{\alpha}}=0
$$

As we have already mentioned all the equations that arise from the Killing spinor equations are linear in the fluxes, geometry and the first derivatives of the functions $f, g_{1}$ and $g_{2}$. Although, this linear system may appear rather involved it can be solved to express the fluxes in terms of the geometry and to find the conditions on the geometry of the spacetime required by the existence of an $S U(4) \ltimes \mathbb{R}^{8}$ invariant Killing spinor. We remark that we have not used the self-duality condition on the five-form flux $F$ in the above conditions. However, the self-duality condition will be implemented in the solution of the linear system in the appendices.

\subsection{Geometry of spacetime}

\subsubsection{Conditions on the geometry}

The expressions of the fluxes in terms of the geometry that solve the linear system of the previous section and the self-duality condition for $F$ can be found in appendix B. Here, we summarize the conditions we have found on the geometry of the spacetime 
for backgrounds that admit an $S U(4) \ltimes \mathbb{R}^{8}$ invariant Killing spinor. In this case, it is assumed that the functions $f, g_{1}, g_{2}$ of the Killing spinor are generic, i.e. that they do not satisfy any relations. The conditions on the geometry that arise from the solution of the Killing spinor equations are

$$
\begin{gathered}
\Omega_{+,+\alpha}=\Omega_{\alpha,+}{ }^{\alpha}=-\Omega_{\bar{\alpha},+}{ }^{\bar{\alpha}}=\Omega_{+, \alpha}{ }^{\alpha}+\frac{i g_{2}}{f} Q_{+}=0, \\
\Omega_{\alpha,+\beta}=0, \quad \Omega_{\alpha,+\bar{\beta}}+\Omega_{\bar{\beta},+\alpha}=0 \\
2 \partial_{+} f+\Omega_{+,-+} f+Q_{+} g_{1}=0, \quad 2 \partial_{+} g_{1}+\Omega_{+,-+} g_{1}-\frac{f^{2}-g_{2}^{2}}{f} Q_{+}=0, \\
2 \partial_{+} g_{2}+\Omega_{+,-+} g_{2}-\frac{g_{1} g_{2}}{f} Q_{+}=0, \\
\partial_{-}\left(f^{2}+g_{2}^{2}+g_{1}^{2}\right)+\Omega_{-,-+}\left(f^{2}+g_{2}^{2}+g_{1}^{2}\right)=0, \\
\partial_{\bar{\alpha}}\left(f^{2}+g_{1}^{2}+g_{2}^{2}\right)+\left(\Omega_{\bar{\alpha},-+}+\Omega_{-, \bar{\alpha}+}\right)\left(f^{2}+g_{1}^{2}+g_{2}^{2}\right)=0 \\
\Omega_{+, \bar{\alpha} \bar{\beta}}=0 .
\end{gathered}
$$

Observe that the conditions (3.28) imply that

$$
\partial_{+}\left(f^{2}+g_{1}^{2}+g_{2}^{2}\right)+\Omega_{+,-+}\left(f^{2}+g_{1}^{2}+g_{2}^{2}\right)=0 .
$$

This condition is used later to show that the spacetime admits a Killing vector field. Observe also that the first equality in the second equation in (3.26) is not independent but follows from the second equation in (3.27). The last equation (3.31) relates two components of the Levi-Civita connection. In what follows, we shall focus on the conditions (3.26)-(3.30). Note that $P$ determines the scalars up to a $U(1)$ gauge choice. So $Q$ is specified up to a $U(1)$ gauge transformation. Therefore one of the equations in (3.28) can be used to determine $Q_{+}$. However, one can also view them as conditions on the geometry.

\subsubsection{Spacetime forms and the geometry of spacetime}

One way to analyze the conditions (3.26)-(3.30) is to find the spacetime forms that arise from spinor bi-linears and are associated to the Killing spinor $\epsilon$. Unlike the case of elevendimensional supergravity, the Killing spinor $\epsilon=\frac{1}{\sqrt{2}}\left[\left(f-g_{2}+i g_{1}\right) 1+\left(f+g_{2}+i g_{1}\right) e_{1234}\right]$ is complex ${ }^{10}$. Because of this, certain $\operatorname{Pin}(9,1)$ and $\operatorname{Spin}(9,1)$ invariant operators ${ }^{11}$ act

\footnotetext{
${ }^{10}$ We have normalized the Killing spinor with an additional factor of $1 / \sqrt{2}$.

${ }^{11}$ Such operators are $L_{ \pm}$of appendix A.
} 
non-trivially on $\epsilon$ and give other spinors on the spacetime independent from $\epsilon$. It turns out that it suffices ${ }^{12}$ to consider the spinor

$$
\tilde{\epsilon}=C^{*} \epsilon=\frac{1}{\sqrt{2}}\left[\left(f+g_{2}-i g_{1}\right) 1+\left(f-g_{2}-i g_{1}\right) e_{1234}\right]
$$

The spinor $\tilde{\epsilon}$ may not be a Killing spinor. The spacetime forms bi-linears associated with the pairs $(\epsilon, \epsilon),(\epsilon, \tilde{\epsilon})$ and $(\tilde{\epsilon}, \tilde{\epsilon})$ can be easily computed using the results of appendix A. In particular, we find three one-forms

$$
\begin{aligned}
& \kappa(\epsilon, \epsilon)=\left[\left(f+i g_{1}\right)^{2}-g_{2}^{2}\right]\left(e^{0}-e^{5}\right), \quad \kappa(\tilde{\epsilon}, \tilde{\epsilon})=\left[\left(f-i g_{1}\right)^{2}-g_{2}^{2}\right]\left(e^{0}-e^{5}\right), \\
& \kappa(\epsilon, \tilde{\epsilon})=\left[f^{2}+g_{1}^{2}+g_{2}^{2}\right]\left(e^{0}-e^{5}\right),
\end{aligned}
$$

a three-form

$$
\xi(\epsilon, \tilde{\epsilon})=2 i f g_{2}\left(e^{0}-e^{5}\right) \wedge \omega
$$

and three five-forms

$$
\begin{aligned}
\tau(\epsilon, \epsilon)= & \frac{1}{2}\left(f-g_{2}+i g_{1}\right)^{2}\left(e^{0}-e^{5}\right) \wedge \chi+\frac{1}{2}\left(f+g_{2}+i g_{1}\right)^{2}\left(e^{0}-e^{5}\right) \wedge \chi^{*} \\
& -\frac{1}{2}\left[\left(f+i g_{1}\right)^{2}-g_{2}^{2}\right]\left(e^{0}-e^{5}\right) \wedge \omega \wedge \omega, \\
\tau(\tilde{\epsilon}, \tilde{\epsilon})= & \frac{1}{2}\left(f+g_{2}-i g_{1}\right)^{2}\left(e^{0}-e^{5}\right) \wedge \chi+\frac{1}{2}\left(f-g_{2}-i g_{1}\right)^{2}\left(e^{0}-e^{5}\right) \wedge \chi^{*} \\
& -\frac{1}{2}\left[\left(f-i g_{1}\right)^{2}-g_{2}^{2}\right]\left(e^{0}-e^{5}\right) \wedge \omega \wedge \omega, \\
\tau(\epsilon, \tilde{\epsilon})= & \left(f^{2}-g_{2}^{2}+g_{1}^{2}\right)\left(e^{0}-e^{5}\right) \wedge \operatorname{Re} \chi-2 g_{1} g_{2}\left(e^{0}-e^{5}\right) \wedge \operatorname{Im} \chi \\
& -\frac{1}{2}\left[f^{2}+g_{1}^{2}+g_{2}^{2}\right]\left(e^{0}-e^{5}\right) \wedge \omega \wedge \omega .
\end{aligned}
$$

Observe that the one-forms are along the same direction but this is not the case for the five-forms. The three-form vanishes if $g_{2}=0$ and the $S U(4)$ structure enhances to a $\operatorname{Spin}(7)$ structure. The relations between the various forms are apparent from their formulae. For example taking the inner product of the three-form with respect to one of the one-forms, one gets a two-form on the spacetime which after an appropriate normalization can be interpreted as the Kähler form of an eight-dimensional subspace.

The spacetime Killing spinor form bi-linears above of supersymmetric IIB backgrounds are complex, unlike those of eleven-dimensional supergravity which are real. This is not surprising because both $\epsilon$ and $\tilde{\epsilon}$ are complex. It appears that the geometry of supersymmetric IIB backgrounds is complex. So the interpretation of the various geometric condition that arise from supersymmetry for IIB backgrounds may require to complexify the tangent bundle and the bundle of forms of the spacetime.

To investigate further the geometry of spacetime, we introduce a frame such that

$$
d s^{2}=2 e^{+} e^{-}+2 \delta_{\alpha \bar{\beta}} e^{\alpha} e^{\bar{\beta}},
$$

\footnotetext{
${ }^{12}$ Because the spinor $\epsilon$ is Weyl the other operator does not give a new independent spinor.
} 
where $e^{-}=1 / \sqrt{2}\left(-e^{0}+e^{5}\right), e^{+}=1 / \sqrt{2}\left(e^{0}+e^{5}\right)$ and $e^{\alpha}, e^{\bar{\alpha}}$ is a Hermitian frame. After some rescaling, the third one-form in (3.34) can be written as

$$
\kappa=-\frac{1}{\sqrt{2}} \kappa(\epsilon, \tilde{\epsilon})=\left(f^{2}+g_{1}^{2}+g_{2}^{2}\right) e^{-} .
$$

We denote the associated null vector field by $X$, i.e. $X=\left(f^{2}+g_{1}^{2}+g_{2}^{2}\right) e_{+}$, where $e^{A}\left(e_{B}\right)=\delta_{B}^{A}$ and $e_{B}$ is the coframe. Using the first equation in (3.26) and (3.32), one can show that $X$ is self-parallel, i.e. it satisfies the equation

$$
X^{B} \nabla_{B} X^{A}=0
$$

In addition $X$ is Killing, $\nabla_{A} X_{B}+\nabla_{B} X_{A}=0$. This follows from the equations (3.28)(3.30) and (3.32). We have not found an interpretation for all the conditions in (3.26). But some of them imply

$$
X^{A} \nabla_{A} \chi_{B_{1} \ldots B_{4}}\left(\chi^{*}\right)^{B_{1} \ldots B_{4}}=0
$$

The $S U(4)$ structure on the spacetime is generic. If there were a restriction on it, there must have been a relation in (3.26)-(3.31) that restricted the components of the LeviCivita connection $\Omega$ along the $S U(4)$ directions $e^{\alpha}, e^{\bar{\alpha}}$. But there is no such relation.

One can introduce coordinates adapted to the null, Killing vector field $X, X=\frac{\partial}{\partial u}$ and write the spacetime metric as

$$
d s^{2}=2\left(W d v+m_{I} d y^{I}\right)\left(U d u+V d v+n_{I} d y^{I}\right)+\gamma_{I J} d y^{I} d y^{J}
$$

where $W, U, V, m_{I}, n_{I}$ and $\gamma_{I J}$ are functions of all coordinates, $I, J=1, \ldots, 8$. Since $X$ is Killing, all the components of the metric are independent of $u$. In addition $U=$ $f^{2}+g_{1}^{2}+g_{2}^{2}$. To see this, we introduce the frame

$$
e^{-}=W d v+m_{I} d y^{I}, \quad e^{+}=U d u+V d v+n_{I} d y^{I}, \quad e^{i}=e_{J}^{i} d y^{J},
$$

where $\gamma_{I J}=\delta_{i j} e_{I}^{i} e_{J}^{j} d y^{I} d y^{J}$. Then we have

$$
X=\left(f^{2}+g_{1}^{2}+g_{2}^{2}\right) e_{+}=\frac{\partial}{\partial u}
$$

where $e_{B}$ is

$$
\begin{array}{r}
e_{+}=U^{-1} \frac{\partial}{\partial u}, \quad e_{-}=W^{-1} \frac{\partial}{\partial v}-(U W)^{-1} V \frac{\partial}{\partial u} \\
e_{i}=e_{i}^{J} \frac{\partial}{\partial y^{J}}+\left(-\frac{n_{i}}{U}+\frac{V m_{i}}{W U}\right) \frac{\partial}{\partial u}-\frac{m_{i}}{W} \frac{\partial}{\partial v}
\end{array}
$$

where $e_{I}^{i} e_{j}^{I}=\delta^{i}{ }_{j}, m_{i}=m_{I} e_{i}^{I}$ and $n_{i}=n_{I} e_{i}^{I}$. However, the Killing vector field can be written as

$$
X=\left(f^{2}+g_{1}^{2}+g_{2}^{2}\right) e_{+}=\left(f^{2}+g_{1}^{2}+g_{2}^{2}\right) U^{-1} \frac{\partial}{\partial u}=\frac{\partial}{\partial u}
$$


Therefore $U=f^{2}+g_{1}^{2}+g_{2}^{2}$.

To write the metric in (3.41), we have separated a coordinate $v$. Unlike $u$, there is no natural way to choose the coordinate $v$, i.e. the conditions on the geometry (3.26)-(3.31) implied by the Killing spinor equations do not lead to a definition of $v$. Because of this, there is no natural way to define an eight-dimensional submanifold $\Sigma$ in the spacetime which can be identified as that that has an $S U(4)$ structure.

A simplification of the conditions on the geometry (3.29) and (3.30), and of the local expression of the metric can be made by fixing a gauge for the transformation (3.1) that scales the Killing spinor $\epsilon$ with a positive spacetime function ${ }^{13}$. For example, one can fix this gauge freedom by setting

$$
U=f^{2}+g_{1}^{2}+g_{2}^{2}=1
$$

In this gauge (3.29) and (3.30) imply that $\Omega_{-,-+}=0$ and $\Omega_{\bar{\alpha},-+}=-\Omega_{-, \bar{\alpha}+}$. In addition, one can set $U=1$ in (3.41), (3.42) and (3.44). In turn, (3.28) implies that $Q_{+}=$ $-2 g_{1}^{-1} \partial_{+} f$ and $\partial_{+}\left(f g_{2}\right)=0$.

Examples of spacetimes that have the structure we have investigated in this section are Lorentzian extensions of one-parameter families of a manifolds with a generic $S U(4)$ structure. The metric on such a family can be written as

$$
d \hat{s}^{2}=2 B^{2} d v^{2}+\hat{\gamma}_{I J}\left(d y^{I}+A^{I} d v\right)\left(d y^{J}+A^{J} d v\right)
$$

where $B, \gamma_{I J}$ and $A$ depend on the coordinates $v, y^{I}, I=1, \ldots, 8$. The component $A$ can be thought of as a non-linear connection of the family. Setting $u=$ const in (3.41), we find that

$$
\begin{aligned}
d \tilde{s}^{2} & =2\left(W d v+m_{I} d y^{I}\right)\left(V d v+n_{I} d y^{I}\right)+\gamma_{I J} d y^{I} d y^{J} \\
& =2 U V d v^{2}+2\left(W n_{I}+V m_{I}\right) d v d y^{I}+\left(\gamma_{I J}+m_{I} n_{J}\right) d y^{I} d y^{J}
\end{aligned}
$$

Comparing this with (3.47), we get

$$
\begin{gathered}
U V=B^{2}+\frac{1}{2} \hat{\gamma}_{I J} A^{I} A^{J} \\
W n_{I}+V m_{I}=\hat{\gamma}_{I J} A^{J} \\
\gamma_{I J}+m_{(I} n_{J)}=\hat{\gamma}_{I J}
\end{gathered}
$$

It is clear that to specify the geometry of spacetime additional data are needed which determine the extension of the $S U(4)$ family to a Lorentian manifold. In turn these are related to the type of reduction of the $\operatorname{Spin}(9,1)$ structure to the $S U(4) \ltimes \mathbb{R}^{8}$ structure of ten-dimensional spacetime.

\subsection{A null geodesic congruence}

An alternative way to interpret the conditions on the geometry of the spacetime (3.26)(3.31) is to use geodesic congruences. Since $X$ is null and self-parallel, it defines a null

\footnotetext{
${ }^{13}$ Further gauge fixing is possible. For example, one can use $R_{++, A B}=0$ to set $\Omega_{+, A B}=0$.
} 
geodesic congruence in the spacetime. In addition $X$ is Killing. This implies that the null geodesic congruence is divergence free, i.e.

$$
\nabla_{A} X^{A}=0
$$

and shear free ${ }^{14}$, i.e.

$$
s_{A B}=\nabla_{A} X_{B}+\nabla_{B} X_{A}-\frac{2}{9} \theta\left(g_{A B}-X_{A} X_{B}\right)=0 .
$$

However, the geodesic congruence is not rotation free, i.e.

$$
r_{A B}=\nabla_{A} X_{B}-\nabla_{B} X_{A}
$$

does not vanish. Because of this, one cannot introduce Penrose coordinates on the spacetime [30] along the null geodesic congruence defined by $X$. In particular, this implies that one cannot define a coordinate $v$. Nevertheless, if one choose a null geodesic in the null geodesic congruence defined by $X$, it is always possible to embed it in a rotation free null geodesic congruence and then take the associated Penrose limit. It turns out that the plane wave at the limit is Minkowski space because $X$ is Killing and so the plane wave metric in Rosen coordinates does not depend on the affine parameter of the geodesic, see [31].

\section{$4 \operatorname{Spin}(7) \ltimes \mathbb{R}^{8}$-invariant Killing spinors}

\subsection{The conditions on the geometry}

The most general IIB Killing spinor which is invariant under $\operatorname{Spin}(7) \ltimes \mathbb{R}^{8}$ is $\epsilon=(f+$ $i g)\left(1+e_{1234}\right)$, where $f, g$ are real functions. It is always possible to choose a gauge such that $\epsilon=\left(1+e_{1234}\right)$. This is because the the Killing spinor equations of IIB supergravity are covariant under the transformation

$$
e^{w \Gamma^{-+}+i \varphi}
$$

after an appropriate rotation of $G$. The parameters $w$ and $\varphi$ can be chosen such that $g=0$ and $f=1$. However for many computations it is useful to have the freedom to rotate with $e^{i \varphi}$. Because of this we shall allow $f, g$ to be arbitrary. However as we shall explain later the $e^{w \Gamma^{-+}}$transformation can be used to simplify the conditions on the geometry of spacetime. One can choose either $f \neq 0$ or $g \neq 0$. In what follows we shall take $f \neq 0$. The conditions on the geometry and fluxes that arise from the Killing spinor equations for $\epsilon=(f+i g)\left(1+e_{1234}\right)$ can be easily derived from those of an $S U(4) \ltimes \mathbb{R}^{8}$ invariant spinor by setting $f=f, g_{1}=g$ and $g_{2}=0$ in the conditions of section (3.1). Because of this, we shall not state the conditions for supersymmetry again. Instead, we summarize the conditions for the geometry. The solution of the Killing spinor equations

\footnotetext{
${ }^{14}$ If $X$ is a timelike or spacelike vector, then the last term in the formulae below is weighted by the length square of $X$ such that $s_{A}{ }^{A}=0$.
} 
and the expressions of the fluxes in terms of the geometry are given in appendix $\mathrm{C}$. The expressions for the fluxes have been simplified using the self-duality of $F$.

The conditions on the spacetime geometry for backgrounds that admit the $\operatorname{Spin}(7) \ltimes$ $\mathbb{R}^{8}$ invariant Killing spinor $\epsilon=(f+i g)\left(1+e_{1234}\right)$ are

$$
\begin{gathered}
\Omega_{+, \alpha+}=\Omega_{\alpha,+}{ }^{\alpha}=\Omega_{+, \alpha}{ }^{\alpha}=0, \\
\Omega_{\alpha,+\bar{\beta}}+\Omega_{\bar{\beta},+\alpha}=0, \quad \Omega_{\alpha,+\beta}+\Omega_{\beta,+\alpha}=0, \\
2 \partial_{+} f+\Omega_{+,-+} f+Q_{+} g=0, \quad 2 \partial_{+} g+\Omega_{+,-+} g-Q_{+} f=0, \\
\partial_{-}\left(f^{2}+g^{2}\right)+\Omega_{-,-+}\left(f^{2}+g^{2}\right)=0, \\
\partial_{\bar{\alpha}}\left(f^{2}+g^{2}\right)+\left(\Omega_{\bar{\alpha},-+}+\Omega_{-, \bar{\alpha}+}\right)\left(f^{2}+g^{2}\right)=0, \\
\Omega_{\alpha,+\beta}-\frac{1}{2} \epsilon_{\alpha \beta}{ }^{\bar{\gamma}_{1} \bar{\gamma}_{2}} \Omega_{\bar{\gamma}_{1},+\bar{\gamma}_{2}}=0, \\
\Omega_{+, \bar{\alpha} \bar{\beta}}-\frac{1}{2} \epsilon_{\bar{\alpha} \bar{\beta}}{ }^{\gamma_{1} \gamma_{2}} \Omega_{+, \gamma_{1} \gamma_{2}}=0 .
\end{gathered}
$$

The conditions that we have found for the geometry of supersymmetric backgrounds with a $\operatorname{Spin}(7) \ltimes \mathbb{R}^{8}$ invariant Killing spinor are closely related to those that we have derived for backgrounds with an $S U(4) \ltimes \mathbb{R}^{8}$ invariant Killing spinor. However there are some differences. For example the second equation in (4.2) is different from the first equation in (3.27). The rest of the equations in (4.2)-(4.5) are related to (3.27)-(3.30) after setting $g_{2}=0$. In addition (3.26) is same as in (4.1) after setting $g_{2}=0$. The equations (4.6) and (4.7) differ from (3.31) and the second equation in (4.2).

\subsection{The geometry of spacetime}

To interpret some of the geometric conditions (4.1)-(4.7) that we have derived from the Killing spinor equations, we shall use the spacetime form bi-linears. The $\operatorname{Spin}(7) \ltimes \mathbb{R}^{8}$ invariant Killing spinor ${ }^{15} \epsilon=\frac{1}{\sqrt{2}}(f+i g)\left(1+e_{1234}\right)$ is complex. Because of this, as we have explained in the case of an $S U(4) \ltimes \mathbb{R}^{8}$ invariant Killing spinor, we should consider the spacetime forms associated with the pairs $(\epsilon, \epsilon),(\epsilon, \tilde{\epsilon})$ and $(\tilde{\epsilon}, \tilde{\epsilon})$, where $\tilde{\epsilon}=C * \epsilon=\frac{1}{\sqrt{2}}(f-i g)\left(1+e_{1234}\right)$ and $C$ is the charge conjugation matrix, see appendix A. We can easily compute these spacetime forms using the formulae that we have collected in appendix A. In particular, we find three one-forms

$$
\begin{aligned}
& \kappa(\epsilon, \epsilon)=(f+i g)^{2}\left(e^{0}-e^{5}\right), \quad \kappa(\tilde{\epsilon}, \tilde{\epsilon})=(f-i g)^{2}\left(e^{0}-e^{5}\right) \\
& \kappa(\epsilon, \tilde{\epsilon})=\left(f^{2}+g^{2}\right)\left(e^{0}-e^{5}\right),
\end{aligned}
$$

\footnotetext{
${ }^{15}$ We have normalized the spinor with an additional factor of $1 / \sqrt{2}$.
} 
and three five-forms

$$
\begin{aligned}
& \tau(\epsilon, \epsilon)=(f+i g)^{2}\left(e^{0}-e^{5}\right) \wedge \phi, \quad \tau(\tilde{\epsilon}, \tilde{\epsilon})=(f-i g)^{2}\left(e^{0}-e^{5}\right) \wedge \phi \\
& \tau(\epsilon, \tilde{\epsilon})=\left(f^{2}+g^{2}\right)\left(e^{0}-e^{5}\right) \wedge \phi,
\end{aligned}
$$

where

$$
\phi=\operatorname{Re} \chi-\frac{1}{2} \omega \wedge \omega
$$

It is easy to recognize from the expressions for $\chi$ and $\omega$ in appendix A that $\phi$ is the usual $\operatorname{Spin}(7)$-invariant four-form. Some of the one-forms and five-forms above are complex as may have been expected because the spinors $\epsilon$ and $\tilde{\epsilon}$ are complex. Unlike the $S U(4) \ltimes \mathbb{R}^{8}$ invariant case, the three-form bi-linears vanish. This may have been expected because there is no apparent $\operatorname{Spin}(7)$ invariant three-form which can be constructed on a tendimensional manifold.

Writing the metric as (3.37) by introducing the frame $e^{+}, e^{-}, e^{\alpha}, e^{\bar{\alpha}}$, we can write the third one-form bi-linear as $\kappa=-\frac{1}{\sqrt{2}} \kappa(\epsilon, \tilde{\epsilon})=\left(f^{2}+g^{2}\right) e^{-}$. The associated vector field is $X=\left(f^{2}+g^{2}\right) e_{+}$, where $e^{A}\left(e_{B}\right)=\delta^{A}{ }_{B}$. As in the $S U(4) \ltimes \mathbb{R}^{8}$ case, the first condition in (4.1) and the conditions (4.2)-(4.5) imply that the null vector field $X$ is self-parallel and Killing. Therefore $X$ defines a divergence and shear free null geodesic congruence. One can adapt coordinates along $X$ and write the spacetime metric as in (3.41) with $U=f^{2}+g^{2}$, see [4] for a similar coordinate system on the spacetime of eleven-dimensional backgrounds with a null Killing spinor. The construction of a local expression for the metric and the introduction of a local frame on the spacetime can be done as in the $S U(4) \ltimes \mathbb{R}^{8}$ case that we have presented in section three. Because of this, we shall not repeat the construction here. The $\operatorname{Spin}(7)$ structure on the spacetime is generic. There are no relations between the components of the metric that lie along the $\left(e^{\alpha}, e^{\bar{\alpha}}\right)$ directions.

One can use the transformation that scales the Killing spinor $\epsilon$ with a positive spacetime function, as in (3.1), to set $f^{2}+g^{2}=1$. Then (4.4) and (4.5) imply that $\Omega_{-,-+}=0$ and $\Omega_{-, \alpha+}=-\Omega_{\alpha,-+}$. In adition, (4.3) gives $Q_{+}=-2 g^{-1} \partial_{+} f$.

It remains to interpret the second condition in (4.1), and the conditions (4.6) and (4.7). These can be combined. In particular they imply that $\Omega_{+, i j}$ and $\Omega_{[i, j]+}$ lie in the Lie algebra of $\operatorname{Spin}(7)$, or equivalently (4.1) (4.6) and (4.7) imply that

$$
\left.\Omega_{+, i j}\right|_{\Lambda_{7}^{2}\left(\mathbb{R}^{8}\right)}=\left.\Omega_{[i, j]+}\right|_{\Lambda_{7}^{2}\left(\mathbb{R}^{8}\right)}=0,
$$

where we have used the decomposition of $\Lambda^{2}\left(\mathbb{R}^{8}\right)=\operatorname{spin}(7) \oplus \Lambda_{7}^{2}\left(\mathbb{R}^{8}\right)$ under $\operatorname{Spin}(7)$.

Examples of spacetimes with the above structure are Lorentzian extensions of oneparameter families of eight-dimensional manifolds with generic $\operatorname{Spin}(7)$ structures. The relation between the metric on the family and that of spacetime can be described as for the $S U(4) \ltimes \mathbb{R}^{8}$ case which has been presented in section three. 


\section{$5 \quad$ Pure spinors as Killing spinors}

\subsection{Conditions on the geometry}

A special class of supersymmetric backgrounds with an $S U(4) \ltimes \mathbb{R}^{8}$ invariant Killing spinor is that for which the Killing spinor is in addition pure. As we have mentioned in the introduction, there are different definitions of what a pure spinor is. Here we adopt the definition that a pure spinor is that for which the associated one-form bi-linear vanishes. Applying this definition to the $S U(4) \ltimes \mathbb{R}^{8}$ invariant spinor $\eta=a 1+b e_{1234}$, $a, b \in \mathbb{C}$, it can be shown using the results in appendix A, that $\eta$ is pure iff $a b=0$. In particular for the $S U(4) \ltimes \mathbb{R}^{8}$ invariant Killing spinor $\epsilon$, we find

$$
\left(f+i g_{1}\right)^{2}-g_{2}^{2}=0
$$

which in turn gives

$$
g_{1}=0, \quad f^{2}=g_{2}^{2}
$$

i.e. $g_{1}=0$ and $f= \pm g_{2}$.

First consider the case, $g_{1}=0$ and $f=-g_{2}$. The pure spinor ${ }^{16}$ is

$$
\epsilon=h 1,
$$

where $h=2 f$. To derive the conditions required for $\epsilon=h 1$ to be a Killing spinor, we simply set $g_{1}=0$ and $f=-g_{2}$ in the conditions of section (3.1) that we have derived for the existence of an $S U(4) \ltimes \mathbb{R}^{8}$ invariant Killing spinor. Because of this, we shall not state these conditions again. Instead, we summarize the conditions on the geometry that are required for $h 1$ to be a Killing spinor. The derivation of these formulae can be found in appendix D.

The conditions on the geometry of a background to admit $\epsilon=h 1$ as Killing spinor are

$$
\begin{gathered}
\Omega_{+,+\alpha}=\Omega_{\alpha,+}{ }^{\alpha}=-\Omega_{\bar{\alpha},+}{ }^{\bar{\alpha}}=-\Omega_{+, \alpha}{ }^{\alpha}+i Q_{+}=0, \\
\Omega_{\alpha,+\bar{\beta}}+\Omega_{\bar{\beta},+\alpha}=0, \quad \Omega_{\alpha,+\beta}=0, \\
2 \partial_{+} \log h+\Omega_{+,-+}=0, \quad 2 \partial_{-} \log h+\Omega_{-,-+}=0, \\
2 \partial_{\bar{\alpha}} \log h+\Omega_{\bar{\alpha},-+}+\Omega_{-, \bar{\alpha}+}=0, \\
\Omega_{+, \alpha \beta}=0, \\
i Q_{\bar{\alpha}}-2 \Omega_{-,+\bar{\alpha}}-\Omega_{\bar{\alpha}, \beta}{ }^{\beta}+\Omega_{\beta, \bar{\alpha}}{ }^{\beta}=0 .
\end{gathered}
$$

\footnotetext{
${ }^{16}$ Observe that the spinor 1 is annihilated by half of the gamma matrices so it is a pure spinor according to the other definition that has been mentioned in the introduction.
} 
As expected these conditions on the geometry resemble those of the generic $S U(4) \ltimes \mathbb{R}^{8}$ invariant spinor in (3.26)-(3.31). The simplification in the above conditions appears because the Killing spinor $h 1$ depends on one function while the generic $S U(4) \ltimes \mathbb{R}^{8}$ invariant spinor depends on three functions. The expressions for the fluxes in terms of the geometry are summarized in appendix D. The main difference between the pure spinor case and the generic $S U(4) \ltimes \mathbb{R}^{8}$ case is the condition (5.8). This condition can be rewritten as

$$
Q_{j} J_{i}^{j}+2 \Omega_{-,+i}+\left(W_{5}\right)_{i}=0
$$

where $\left.W_{5}=\chi\right\lrcorner d \chi$ is a Gray-Hervella class. However this equation is valid only if it is restricted along the $e^{i}$ frame directions as indicated.

Next consider the other pure spinor $k e_{1234}$. Again, the conditions that arise from the Killing spinor equations for $k e_{1234}$ to be Killing spinor can be derived from those we have derived for case of a generic $S U(4) \ltimes \mathbb{R}^{8}$ invariant spinor. For this, we set $g_{1}=0$ and $h=2 f=2 g_{2}$ in the conditions of section (3.1). We shall not present here the derivation of the solution to the resulting equations. This can be found in appendix E. It turns out that the conditions on the geometry of a background to admit $\epsilon=k e_{1234}$ as Killing spinor are the same as those given for the spinor $h 1$ (5.4)-(5.7) after replacing the function $h$ with the function $k$, expect for the sign of $Q_{+}$in (5.4) and in (5.8). The expressions for the fluxes in terms of the geometry are given in appendix E.

\subsection{Geometry and spacetime forms}

We examine the geometry of a spacetime with Killing spinor $h 1$. The analysis for the Killing spinor $k e_{1234}$ is similar and it will be omitted. Since $h 1$ is a pure spinor, the associated one-form bi-linear vanishes. But as we have explained, if $\epsilon$ is defined on the spacetime, then so is $\tilde{\epsilon}=C *(\epsilon)=h e_{1234}$ even though that it may not be a Killing spinor. The spacetime form bi-linears associated with the pairs o $(\epsilon, \epsilon),(\epsilon, \tilde{\epsilon})$ and $(\tilde{\epsilon}, \tilde{\epsilon})$ are a one-form

$$
\kappa(\epsilon, \tilde{\epsilon})=h^{2}\left(e^{0}-e^{5}\right)
$$

a three-form

$$
\xi(\epsilon, \tilde{\epsilon})=-i h^{2}\left(e^{0}-e^{5}\right) \wedge \omega
$$

and three five-forms

$$
\begin{aligned}
\tau(\epsilon, \epsilon) & =h^{2}\left(e^{0}-e^{5}\right) \wedge \chi, \quad \tau(\tilde{\epsilon}, \tilde{\epsilon})=h^{2}\left(e^{0}-e^{5}\right) \wedge \chi^{*}, \\
\tau(\epsilon, \tilde{\epsilon}) & =-\frac{h^{2}}{2}\left(e^{0}-e^{5}\right) \wedge \omega \wedge \omega .
\end{aligned}
$$

We introduce the frame $e^{+}, e^{-}, e^{\alpha}, e^{\bar{\alpha}}$ and write the metric as in (3.37). After some rescaling, the one-form can be written as

$$
\kappa=-\frac{1}{\sqrt{2}} \kappa(\epsilon, \tilde{\epsilon})=h^{2} e^{-} .
$$


The associated null vector field with $X$ is self-parallel and Killing. The geometry of the spacetime is similar to that described for the generic $S U(4) \ltimes \mathbb{R}^{8}$ invariant spinor. For example the metric in local coordinates is given as in (3.41) with $U=h^{2}$. In the pure spinor case, one also has the condition (5.8). If the spacetime is constructed as an one parameter family of manifolds, then the $S U(4)$ structure of the eight-dimensional manifolds is restricted by this condition.

\section{Backgrounds with two pure Killing spinors}

\subsection{Geometry and fluxes}

Combining the results we present in section five and in appendices D and E, we shall give the conditions on the geometry and the fluxes implied by the Killing spinor equations for supersymmetric backgrounds with Killing spinors $\epsilon=h 1$ and $\eta=k e_{1234}$. Thus these backgrounds admit two pure $S U(4) \ltimes \mathbb{R}^{8}$ invariant Killing spinors and they constitute a class of supersymmetric backgrounds with two supersymmetries.

Since the conditions (5.6) are the same for both spinors, it is easy to see that up to an overall constant scale $h=k$. After some computation, the conditions on the geometry required for a background to have Killing spinors $\epsilon=h 1$ and $\eta=h e_{1234}$ are

$$
\begin{gathered}
\Omega_{+,+\alpha}=\Omega_{+, \alpha \beta}=\Omega_{\alpha,+\bar{\beta}}=\Omega_{\alpha,+\beta}=\Omega_{+, \gamma}{ }^{\gamma}=\Omega_{-, \gamma}{ }^{\gamma}=0 \\
2 \partial_{+} \log h+\Omega_{+,-+}=0, \quad 2 \partial_{-} \log h+\Omega_{-,-+}=0 \\
2 \partial_{\bar{\alpha}} \log h+\Omega_{\bar{\alpha},-+}+\Omega_{-, \bar{\alpha}+}=0 \\
\Omega_{\left[\gamma_{1}, \gamma_{2} \gamma_{3}\right]}=0, \quad \Omega_{\bar{\alpha}, \beta}^{\beta}=-\frac{1}{3} \Omega_{\beta}{ }^{\beta}{ }_{\bar{\alpha}}=\Omega_{-,+\bar{\alpha}} \\
\Omega_{\alpha, \bar{\beta}_{1} \bar{\beta}_{2}}-2 \Omega_{-,+\left[\bar{\beta}_{1}\right.} g_{\left.\bar{\beta}_{2}\right] \alpha}=0 .
\end{gathered}
$$

The expressions for the $G$ fluxes in terms of the $F$ fluxes and geometry required by supersymmetry are

$$
\begin{aligned}
& G_{+\alpha \beta}=G_{+\bar{\alpha} \bar{\beta}}=G_{-+\alpha}=G_{-+\bar{\alpha}}=G_{\alpha \beta}^{\beta}=G_{\bar{\alpha} \beta}{ }^{\beta}=G_{+\alpha \bar{\beta}}=0,
\end{aligned}
$$

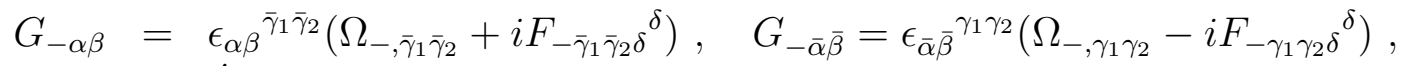

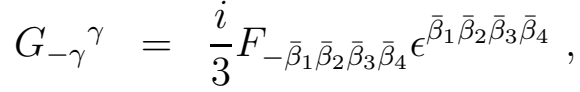

$$
\begin{aligned}
& G_{\alpha \bar{\gamma}_{1} \bar{\gamma}_{2}}=\Omega_{\alpha, \delta_{1} \delta_{2}} \epsilon^{\delta_{1} \delta_{2}} \bar{\gamma}_{1} \bar{\gamma}_{2}, \quad G_{\gamma_{1} \gamma_{2} \bar{\alpha}}=\Omega_{\bar{\alpha}, \bar{\delta}_{1} \bar{\delta}_{2}} \epsilon_{\gamma_{1} \gamma_{2}}^{\bar{\delta}_{1} \bar{\delta}_{2}} \\
& G_{\gamma_{1} \gamma_{2} \gamma_{3}}=4 \Omega_{-,+\bar{\alpha}} \epsilon_{\gamma_{1} \gamma_{2} \gamma_{3}}^{\overline{\bar{a}}}, \quad G_{\bar{\gamma}_{1} \bar{\gamma}_{2} \bar{\gamma}_{3}}=4 \Omega_{-,+\alpha} \epsilon_{\bar{\gamma}_{1} \bar{\gamma}_{2} \bar{\gamma}_{3}}^{\alpha}
\end{aligned}
$$

Similarly, the conditions on the $F$ fluxes are

$$
F_{-+\gamma_{1} \gamma_{2} \gamma_{3}}=F_{+\alpha \beta \gamma \delta}=F_{+\alpha \bar{\beta}_{1} \bar{\beta}_{2} \bar{\beta}_{3}}=F_{+\alpha \bar{\beta} \gamma}{ }^{\gamma}=F_{\alpha \bar{\beta}_{1} \bar{\beta}_{2} \bar{\beta}_{3} \bar{\beta}_{4}}=0
$$




$$
F_{-+\bar{\alpha} \gamma}^{\gamma}=F_{+\alpha}^{\alpha} \delta^{\delta}=F_{-+\alpha \bar{\beta}_{1} \bar{\beta}_{2}}=0, \quad F_{-\gamma}{ }^{\gamma} \delta=2 Q_{-},
$$

and

$$
\epsilon^{\bar{\beta}_{1} \bar{\beta}_{2} \bar{\beta}_{3} \bar{\beta}_{4}} F_{-\bar{\beta}_{1} \bar{\beta}_{2} \bar{\beta}_{3} \bar{\beta}_{4}}=-F_{-\beta_{1} \beta_{2} \beta_{3} \beta_{4}} \epsilon^{\beta_{1} \beta_{2} \beta_{3} \beta_{4}} .
$$

Finally, the conditions on the scalars are

$$
P_{+}=Q_{+}=0, \quad Q_{\alpha}=0, \quad P_{\alpha}=-2 \Omega_{-,+\alpha}, \quad P_{\bar{\alpha}}=-2 \Omega_{-,+\bar{\alpha}} .
$$

The components of the fluxes that do not appear in the above equations are not specified by the Killing spinor equations.

The conditions on the geometry are investigated in the next section. Since the flux $G$ is complex, the two conditions in (6.6) and in (6.7) are independent. In particular, the conditions (6.6) imply that $G_{\alpha \bar{\beta} \bar{\gamma}}$ is the complex conjugate of $G_{\bar{\alpha} \beta \gamma}$. In addition, (6.11) implies that $P_{\alpha}$ is the complex conjugate of $P_{\bar{\alpha}}$.

\subsection{Geometry}

The spacetime forms associated with the Killing spinors $\epsilon=f 1$ and $\eta=h e_{1234}$ have also been given in section 5.2. The vector field $X$ associated to the one-form $\kappa$ (15.13) is a null, self-parallel, Killing vector as for the other supersymmetric backgrounds with $S U(4) \ltimes \mathbb{R}^{8}$ invariant Killing spinors. However, some of the conditions on the geometry in this case are more restrictive. For example $\Omega_{\alpha, \beta+}=0$ vanishes instead of $\Omega_{(\alpha, \beta)+}=0$. Introducing local coordinates for the metric as in (3.41), the conditions $\Omega_{\alpha, \beta+}=\Omega_{\alpha, \bar{\beta}+}=0$ in (6.1) imply that locally $m_{I}=\partial_{I} m$ for some $m=m(v, y)$.

Furthermore, there are restrictions on the $S U(4)$ structure of the ten-dimensional spacetime given in equation (6.3). All the conditions on the geometry we have found can be reexpressed as the vanishing of certain $S U(4) \ltimes \mathbb{R}^{8}$ irreducible representations of the covariant derivative of the spacetime form bi-linears of the Killing spinors as in 32 . We shall not give an exhaustive list because this is just a rewriting of the conditions on the geometry we have already expressed in terms of the spin connection $\Omega$. For example, one can show that $(\nabla \omega)_{[\alpha \beta \gamma]}=0$ implies $\Omega_{[\alpha, \beta \gamma]}=0$.

\section{Conclusions}

We have used the method of [1] to directly solve the Killing spinor equations and the selfduality condition on the five-form field strength of IIB supergravity for one $\operatorname{Spin}(7) \ltimes \mathbb{R}^{8}$ or one $S U(4) \ltimes \mathbb{R}^{8}$ invariant Killing spinor. In both cases, we have found the conditions on the geometry of the spacetime required by supersymmetry. One difference with similar computations in eleven-dimensions [3, 4] is that in IIB supergravity the Killing spinors depend on more than one spacetime function. As an example of our construction, we have presented the solution to the Killing spinor equations for backgrounds that admit one and two pure $S U(4) \ltimes \mathbb{R}^{8}$ invariant Killing spinors. In this case, the Killing spinor equations simplify. Nevertheless, the geometry of the spacetime resembles that of backgrounds with a generic $S U(4) \ltimes \mathbb{R}^{8}$ invariant Killing spinor. There is another class of supersymmetric 
IIB backgrounds with one supersymmetry for which the Killing spinor is $G_{2}$ invariant. We shall present this case elsewhere [21].

We have computed the spacetime forms that are associated to Killing spinor bilinears. It turns out that in general the forms are complex. This is not surprising because the Killing spinors of IIB supergravity are complex. Therefore, the spinorial geometry of IIB backgrounds appears to be associated with complex geometry, i.e. it requires the complexication of the tangent bundle and of the bundle of forms of the spacetime. This is unlike the spinorial geometry of eleven-dimensional backgrounds which is real. In addition, the spacetime forms in both $\operatorname{Spin}(7) \ltimes \mathbb{R}^{8}$ and $S U(4) \ltimes \mathbb{R}^{8}$ cases are related to SLAG and Cayley calibration forms, see e.g. 33. However, in the context of supergravity these forms are not closed and so define generalized calibrations [34, 35]. It seems that IIB supersymmetric backgrounds admit generalized calibrated cycles. Such cycles are the supersymmetric solutions of D-brane worldvolume actions coupled to Ramond-Ramond fields in the absence of $B$ field.

We have seen that the geometry of some supersymmetric IIB backgrounds can be described using the reduction of the structure group from $\operatorname{Spin}(9,1)$ to $S U(4) \ltimes \mathbb{R}^{8}$ and to $\operatorname{Spin}(7) \ltimes \mathbb{R}^{8}$. It is clear that such reductions can be understood in a similar way as that proposed by Gray-Hervella 32 for almost Hermitian manifolds and further developed in [36, 37, 38, see also 39]. However to our knowledge, there is no systematic investigation of the reduction that occurs in the context of IIB supergravity. Supersymmetric IIB backgrounds with more than one Killing spinor may require even more exotic reductions of $\operatorname{Spin}(9,1)$.

\section{Acknowledgements}

The work of U.G. is funded by the Swedish Research Council. This work has been partially supported by the PPARC grant PPA/G/O/2002/00475. J.G. thanks EPSRC for support.

\section{Appendix A Spinors}

The realization of spinors in terms of forms can be found for example in [40, 33]. This has been used in [41] to investigate the parallel spinors and associated forms on special holonomy manifolds. The description of spinors in [40, 33] extends over several chapters and [41] does not describe the construction for Lorentzian manifolds. Because of this, in this appendix, we summarize the essential information needed to realize the spinors of $\operatorname{Spin}(9,1)$ in terms of forms.

Let $V=\mathbb{R}^{9,1}$ be a real vector space equipped with the Lorentzian inner product $<\cdot, \cdot>$. Introduce an orthonormal basis $e_{1}, \ldots, e_{9}, e_{0}, e_{0}$ is along the time direction, and take the subspace $U=\mathbb{R}<e_{1}, \ldots, e_{5}>$ spanned with respect to the first five basis vectors $e_{1}, \ldots, e_{5}$. The space of Dirac spinors is $\Delta_{c}=\Lambda^{*}(U \otimes \mathbb{C})$. This decomposes into two complex chiral representations according to the degree of the form $\Delta_{c}^{+}=\Lambda^{\text {even }}(U \otimes \mathbb{C})$ and $\Delta_{c}^{-}=\Lambda^{\text {odd }}(U \otimes \mathbb{C})$. These are the complex Weyl representations of $\operatorname{Spin}(9,1)$. The 
gamma matrices are represented on $\Delta_{c}$ as

$$
\begin{array}{rlrl}
\Gamma_{0} \eta & \left.\left.=-e_{5} \wedge \eta+e_{5}\right\lrcorner \eta, \quad \Gamma_{5} \eta=e_{5} \wedge \eta+e_{5}\right\lrcorner \eta \\
\Gamma_{i} \eta & \left.=e_{i} \wedge \eta+e_{i}\right\lrcorner \eta, & i=1, \ldots, 4 \\
\Gamma_{5+i} \eta & \left.=i e_{i} \wedge \eta-i e_{i}\right\lrcorner \eta .
\end{array}
$$

The gamma matrices have been chosen such that $\left\{\Gamma_{i} ; i=1, \ldots, 9\right\}$ are Hermitian and $\Gamma_{0}$ is anti-Hermitian with respect to the (auxiliary) inner product

$$
<z^{a} e_{a}, w^{b} e_{b}>=\sum_{a=1}^{5}\left(z^{a}\right)^{*} w^{a},
$$

on $U \otimes \mathbb{C}$ and then extended to $\Delta_{c}$, where $\left(z^{a}\right)^{*}$ is the standard complex conjugate ${ }^{17}$ of $z^{a}$. The above gamma matrices satisfy the Clifford algebra relations $\Gamma_{A} \Gamma_{B}+\Gamma_{B} \Gamma_{A}=2 \eta_{A B}$ with respect to the Lorentzian inner product as expected.

The Dirac inner product on the space of spinors $\Delta_{c}$ is defined as

$$
D(\eta, \theta)=<\Gamma_{0} \eta, \theta>
$$

Observe that while $D$ is invariant under $\operatorname{Spin}(9,1)$ the auxiliary inner product $<$, $>$ is not.

It is known that on even-dimensional manifolds, there are two Spin invariant Majorana inner products. Only one of these inner product is Pin invariant as well. Therefore we expect that there are two $\operatorname{Spin}(9,1)$ invariant Majorana inner products. One of them is defined as

$$
A(\eta, \theta)=<A\left(\eta^{*}\right), \theta>
$$

where the map denoted with the same symbol as the inner product is $A=\Gamma_{12345}$. The other $\operatorname{Spin}(9,1)$ invariant inner product is

$$
B(\eta, \theta)=<B\left(\eta^{*}\right), \theta>
$$

where $B=\Gamma_{06789}$. Observe that the inner product $B$ is in addition Pin invariant and skew-symmetric $B(\eta, \theta)=-B(\theta, \eta)$. The above inner products $A$ and $B$ pair the $\Delta_{c}^{+}$ and $\Delta_{c}^{-}$representations. Moreover, both $\Delta_{c}^{+}$and $\Delta_{c}^{-}$are null with respect to $A$, $B$, i.e. $A, B$ restricted to either $\Delta_{c}^{+}$or $\Delta_{c}^{-}$vanish.

It is well-known that $\operatorname{Spin}(9,1)$ admits two inequivalent Majorana-Weyl representations. So it remains to impose the Majorana condition on the complex Weyl representations we have constructed above. This is done by setting the Dirac conjugate spinor to be equal to the Majorana conjugate one. Equivalently, one can impose the reality condition using an anti-linear map which commutes with the generators of $\operatorname{Spin}(9,1)$ and squares to one. There are two ways of imposing the Majorana-Weyl condition each associated with the two Majorana inner products $A, B$ described above. The associated anti-linear maps are

$$
L_{+}=e^{i \varphi+} \Gamma_{0} A *
$$

\footnotetext{
${ }^{17} \operatorname{In}([1])$ we denote the standard complex of $\eta$ with $\bar{\eta}$ instead of $\eta^{*}$ that we use here.
} 


$$
L_{-}=e^{i \varphi-} \Gamma_{0} B *
$$

The phases in $L_{ \pm}$are arbitrary. Clearly $L_{ \pm}$are antilinear and commute with the generators of $\operatorname{Spin}(9,1)$. The Majorana conditions on the spinors are

$$
L_{ \pm}(\eta)=\eta
$$

These reality conditions map forms of even (odd)-degree to forms of even (odd)-degree and select real subspaces $\Delta_{16}^{+}$and $\Delta_{16}^{-}$in $\Delta_{c}^{+}$and in $\Delta_{c}^{-}$, respectively. These subspaces are the modules of the two inequivalent Majorana-Weyl representations of $\operatorname{Spin}(9,1)$.

In the formulation of IIB supergravity, one can use either the $A\left(L_{+}\right)$or $B\left(L_{-}\right)$ inner product (anti-linear reality map). In this paper, we describe our calculation using the $B$ inner product. The reason for this is that $B$ can be extended to the $\operatorname{Spin}(10,1)$ invariant inner product of eleven-dimensional supergravity. This makes connection with the description of spinor in terms of forms in [11]. In particular the $B$ inner product described in [11] is equivalent up to a change of basis to the inner product $B$ that we have given above. It is also convenient to simply somewhat the Majorana reality condition (A.6). In particular we choose the phase such that

$$
\eta=-\Gamma_{0} B\left(\eta^{*}\right)
$$

or equivalently

$$
\eta^{*}=\Gamma_{6789} \eta
$$

The map $C=\Gamma_{6789}$ is also called charge conjugation matrix, $L_{-}=C *$. Observe that the anti-linear operator $C *$ commutes with the gamma matrices, i.e. $C * \Gamma_{A}=\Gamma_{A} C *$ or equivalently $C^{-1} \Gamma_{A} C=\Gamma_{A}^{*}$. As we have seen, the reality condition can also be expressed as $\eta=C * \eta=C\left(\eta^{*}\right)$.

We shall illustrate the reality condition (A.9) with an example. Consider the complex chiral spinor $a 1+b e_{1234}, a, b \in \mathbb{C}$. The associated real spinor of positive chirality is

$$
\eta=a 1+a^{*} e_{1234}
$$

So we find two real spinors given by $1+e_{1234}$ and $i 1-i e_{1234}$.

It remains to give the spacetime forms associated with pair of spinors $\eta, \theta$. These are

$$
\alpha(\eta, \theta)=\frac{1}{k !} B\left(\eta, \Gamma_{A_{1} \ldots A_{k}} \theta\right) e^{A_{1}} \wedge \ldots \wedge e^{A_{k}}, \quad k=0, \ldots, 9
$$

If both spinors are of the same chirality, then it is sufficient to compute the forms up to degree $k \leq 5$. This is because the forms with degrees $k \geq 6$ are related to those with degrees $k \leq 5$ with a Hodge duality operation. The forms of middle dimension are either self-dual or anti-self-dual.

In many computations that follow it is convenient to use another basis in the space of spinors $\Delta_{c}$. This basis is given in terms of creation and annihilation operators. For this first write

$$
\Gamma_{\bar{\alpha}}=\frac{1}{\sqrt{2}}\left(\Gamma_{\alpha}+i \Gamma_{\alpha+5}\right), \quad \Gamma_{ \pm}=\frac{1}{\sqrt{2}}\left(\Gamma_{5} \pm \Gamma_{0}\right), \quad \Gamma_{\alpha}=\frac{1}{\sqrt{2}}\left(\Gamma_{\alpha}-i \Gamma_{\alpha+5}\right)
$$


Observe that the Clifford algebra relations in the above basis are $\Gamma_{A} \Gamma_{B}+\Gamma_{B} \Gamma_{A}=2 g_{A B}$, where the non-vanishing components of the metric are $g_{\alpha \bar{\beta}}=\delta_{\alpha \bar{\beta}}, g_{+-}=1$. In addition we define $\Gamma^{B}=g^{B A} \Gamma_{A}$. The 1 spinor is a Clifford vacuum, $\Gamma_{\bar{\alpha}} 1=\Gamma_{+} 1=0$ and the representation $\Delta_{c}$ can be constructed by acting on 1 with the creation operators $\Gamma^{\bar{\alpha}}, \Gamma^{+}$ or equivalently any spinor can be written as

$$
\eta=\sum_{k=0}^{5} \frac{1}{k !} \phi_{\bar{a}_{1} \ldots \bar{a}_{k}} \Gamma^{\bar{a}_{1} \ldots \bar{a}_{k}} 1, \quad \bar{a}=\bar{\alpha},+,
$$

i.e. $\Gamma^{\bar{a}_{1} \ldots \bar{a}_{k}} 1$, for $k=0, \ldots, 5$, is a basis in the space of (Dirac) spinors. This is another manifestation of the relation between spinors and forms. See also [42] for other bases of spinors used in the context of supergravity.

\section{A.1 Spacetime forms from spinors}

To compute the spacetime forms that are associated with the $\operatorname{Spin}(7) \ltimes \mathbb{R}^{8}$ - and $S U(4) \ltimes$ $\mathbb{R}^{8}$-invariant spinors, it is sufficient to know the spacetime forms associated with the 1 and $e_{1234}$ spinors. This is because as we have seen 1 and $e_{1234}$ span the $\operatorname{Spin}(7) \ltimes \mathbb{R}^{8}$ and $S U(4) \ltimes \mathbb{R}^{8}$-invariant spinors. As a result, the spacetime forms associated with the $\operatorname{Spin}(7) \ltimes \mathbb{R}^{8}$ - and $S U(4) \ltimes \mathbb{R}^{8}$-invariant spinors are linear combinations of the 1 and $e_{1234}$ spinor form bi-linears. Using (A.11), it is easy to find that the forms associated with the 1 and $e_{1234}$ spinors are the following: A one-form

$$
\kappa\left(e_{1234}, 1\right)=\kappa\left(1, e_{1234}\right)=e^{0}-e^{5},
$$

a three-form

$$
\xi\left(e_{1234}, 1\right)=-\xi\left(1, e_{1234}\right)=i\left(e^{0}-e^{5}\right) \wedge \omega
$$

and five-forms

$$
\begin{array}{r}
\tau(1,1)=\left(e^{0}-e^{5}\right) \wedge \chi \\
\tau\left(e_{1234}, e_{1234}\right)=\left(e^{0}-e^{5}\right) \wedge \chi^{*} \\
\tau\left(e_{1234}, 1\right)=\tau\left(1, e_{1234}\right)=-\frac{1}{2}\left(e^{0}-e^{5}\right) \omega
\end{array}
$$

where

$$
\begin{aligned}
\omega & =e^{1} \wedge e^{6}+e^{2} \wedge e^{7}+e^{3} \wedge e^{8}+e^{4} \wedge e^{9} \\
\chi=\left(e^{1}+i e^{6}\right) & \wedge\left(e^{2}+i e^{7}\right) \wedge\left(e^{3}+i e^{8}\right) \wedge\left(e^{4}+i e^{9}\right) .
\end{aligned}
$$

Note that $\chi$ and $\omega$ are the familiar $S U(4)$ invariant forms.

\section{Appendix B The solution of Killing spinor equa- tions for $S U(4) \ltimes \mathbb{R}^{8}$ - invariant spinors}

\section{B.2 The solution of the linear system}

The independent components of the $P$ and $G$ fluxes are the following:

$$
P_{+}, \quad P_{-}, \quad P_{\alpha}, \quad P_{\bar{\alpha}}
$$




$$
\begin{aligned}
& G_{+-\alpha}, \quad G_{+-\bar{\alpha}}, \quad G_{+\alpha \beta}, \quad G_{+\alpha \bar{\beta}}, \quad G_{+\bar{\alpha} \bar{\beta}} \\
& G_{-\alpha \beta}, \quad G_{-\alpha \bar{\beta}}, \quad G_{-\bar{\alpha} \bar{\beta}}, \quad G_{\alpha \beta \gamma}, \quad G_{\alpha \beta \bar{\gamma}}, \quad G_{\alpha \bar{\beta} \bar{\gamma}}, \quad G_{\bar{\alpha} \bar{\beta} \bar{\gamma}} \text {. }
\end{aligned}
$$

The five-form flux $F$ can be decomposed as

$$
\begin{array}{lllll}
F_{+-\alpha \beta \gamma}, & F_{+-\alpha \beta \bar{\gamma}}, & F_{-\alpha \beta \gamma \delta}, & F_{-\alpha \beta \gamma \bar{\delta}}, & F_{-\alpha \beta \bar{\gamma} \bar{\delta}} \\
F_{+\alpha \beta \gamma \delta}, & F_{+\alpha \beta \gamma \bar{\delta}}, & F_{+\alpha \beta \bar{\gamma} \delta}, & F_{\alpha \beta \gamma \delta \bar{\epsilon}}, & F_{\alpha \beta \gamma \delta \bar{\epsilon}}
\end{array}
$$

up to complex conjugation. However, these components are not independent but they are related by the self-duality condition of $F$. The self-duality condition $F_{M_{1} \ldots M_{5}}=$ $-\frac{1}{5 !} \epsilon_{M_{1} \ldots M_{5}}{ }^{N_{1} \ldots N_{5}} F_{N_{1} \ldots N_{5}}$ relates the above components of $F$ as

$$
\begin{aligned}
F_{\alpha_{1} \alpha_{2} \alpha_{3} \alpha_{4} \bar{\beta}} & =-\frac{1}{6} \epsilon_{\alpha_{1} \alpha_{2} \alpha_{3} \alpha_{4}} \epsilon_{\bar{\beta}}^{\gamma_{1} \gamma_{2} \gamma_{3}} F_{-+\gamma_{1} \gamma_{2} \gamma_{3}} \\
F_{\alpha_{1} \alpha_{2} \alpha_{3} \bar{\beta}_{1} \bar{\beta}_{2}} & =-\frac{1}{2} \epsilon_{\alpha_{1} \alpha_{2} \alpha_{3}}{ }^{\bar{\gamma}_{1}} \epsilon_{\bar{\beta}_{1} \bar{\beta}_{2}}{ }^{\gamma_{2} \gamma_{3}} F_{-+\bar{\gamma}_{1} \gamma_{2} \gamma_{3}} \\
F_{+\alpha_{1} \alpha_{2} \alpha_{3} \alpha_{4}} & =0 \\
F_{+\alpha_{1} \alpha_{2} \alpha_{3} \bar{\beta}} & =-\frac{1}{6} \epsilon_{\bar{\beta}}^{\beta_{1} \beta_{2} \beta_{3}} \epsilon_{\alpha_{1} \alpha_{2} \alpha_{3}}{ }^{\bar{\gamma}} F_{+\beta_{1} \beta_{2} \beta_{3} \bar{\gamma}} \\
F_{+\bar{\beta}_{1} \bar{\beta}_{2} \alpha_{1} \alpha_{2}} & =-\frac{1}{4} \epsilon_{\bar{\beta}_{1} \bar{\beta}_{2}}{ }^{\delta_{1} \delta_{2}} \epsilon_{\alpha_{1} \alpha_{2}}{ }^{\bar{\gamma}_{1} \bar{\gamma}_{2}} F_{+\bar{\gamma}_{1} \bar{\gamma}_{2} \delta_{1} \delta_{2}} \\
F_{-\alpha_{1} \alpha_{2} \alpha_{3} \alpha_{4}} & =\frac{1}{4 !} \epsilon_{\alpha_{1} \alpha_{2} \alpha_{3} \alpha_{4}} \epsilon^{\beta_{1} \beta_{2} \beta_{3} \beta_{4}} F_{-\beta_{1} \beta_{2} \beta_{3} \beta_{4}} \\
F_{-\alpha_{1} \alpha_{2} \alpha_{3} \bar{\beta}} & =\frac{1}{6} \epsilon_{\bar{\beta}}{ }^{\beta_{1} \beta_{2} \beta_{3}} \epsilon_{\alpha_{1} \alpha_{2} \alpha_{3}}{ }^{\bar{\gamma}} F_{-\beta_{1} \beta_{2} \beta_{3} \bar{\gamma}} \\
F_{-\bar{\beta}_{1} \bar{\beta}_{2} \alpha_{1} \alpha_{2}} & =\frac{1}{4} \epsilon_{\bar{\beta}_{1} \bar{\beta}_{2}}{ }^{\delta_{1} \delta_{2}} \epsilon_{\alpha_{1} \alpha_{2}}{ }^{\bar{\gamma}_{1} \bar{\gamma}_{2}} F_{-\bar{\gamma}_{1} \bar{\gamma}_{2} \delta_{1} \delta_{2}} .
\end{aligned}
$$

These imply the following relations

$$
\begin{aligned}
F_{\alpha_{1} \alpha_{2} \alpha_{3} \delta} \delta & =F_{-+\alpha_{1} \alpha_{2} \alpha_{3}} \\
F_{\alpha_{1} \alpha_{2} \bar{\beta} \delta} \delta & =-F_{-+\alpha_{1} \alpha_{2} \bar{\beta}}-2 g_{\bar{\beta}\left[\alpha_{1}\right.} F_{\left.\alpha_{2}\right]-+\delta} \delta \\
F_{\alpha \beta}{ }^{\beta} \gamma & =2 F_{-+\alpha \delta}{ }^{\gamma} \\
F_{+\alpha \beta \delta} \delta & =0 \\
F_{+\alpha_{1} \alpha_{2} \alpha_{3}\left[\bar{\beta} \epsilon_{\bar{\gamma}]} \alpha_{1} \alpha_{2} \alpha_{3}\right.} & =0 \\
F_{+\alpha}{ }^{\alpha}{ }^{\beta} & =0 .
\end{aligned}
$$

Since the $P$ and $G$ are complex fields, the holomorphic and anti-holomorphic components are not complex conjugate, i.e. $P_{\alpha} \neq\left(P_{\bar{\alpha}}\right)^{*}$. Because of this, it is convenient to solve the Killing spinor equations for $G$ and $P$ first. The remaining equations can then be expressed in terms of the spacetime connection $\Omega$, the five-form flux $F$ and the scalar connection $Q$. Since $\Omega, F$ and $Q$ are real, one can analyze the remaining equations using complex conjugation. Throughout this computation, we use the self-duality condition of $F$.

To solve the Killing spinor equations, we assume that the functions $f, g_{1}, g_{2}$ are generic. In particular, we shall take $f \neq \pm g_{2} \neq 0$ and $g_{1} \neq 0$. We shall give the solutions of some special cases in appendices C, D and E. First consider the conditions associated with the algebraic Killing spinor equation. Treating (3.4) and (3.5) as equations for the 
variables $P_{+}$and $G_{+\alpha}{ }^{\alpha}$, observe that the determinant of coefficients is $-\frac{1}{2}\left(f^{2}+g_{1}^{2}+g_{2}^{2}\right)$. So unless the spinor $\epsilon$ vanishes, we find that

$$
P_{+}=G_{+\alpha}{ }^{\alpha}=0
$$

The conditions (3.2) and (3.3) are viewed as equations for $P_{\alpha}$ and $P_{\bar{\alpha}}$ and so both are determined in terms of components of the $G$ flux. From now on, we shall assume that the,$+ \alpha$ and $\bar{\alpha}$-derivatives of the scalars are determined. $P_{-}$remains undetermined by the Killing spinor equations.

Finally, (3.6) is viewed as an equation which relates the $G_{+\alpha \beta}$ and $G_{+\bar{\alpha} \bar{\beta}}$ components of the $G$ flux. It will be used later to express $G_{+\alpha \beta}$ and $G_{+\bar{\alpha} \bar{\beta}}$ in terms of components of the $F$ flux and geometry.

Dualizing (3.16) with respect the epsilon tensor, using (B.6) and taking a trace, we find

$$
\Omega_{\beta,+}^{\beta}=0 .
$$

Substituting (B.7) into (3.22), we find

$$
D_{+}\left(f-g_{2}+i g_{1}\right)+\left[\frac{1}{2} \Omega_{+, \gamma}^{\gamma}+\frac{1}{2} \Omega_{+,-+}\right]\left(f-g_{2}+i g_{1}\right)=0 .
$$

Substituting (B.7) into (3.24) and taking the complex conjugate, we get

$$
D_{+}^{*}\left(f+g_{2}-i g_{1}\right)+\left[\frac{1}{2} \Omega_{+, \gamma}^{\gamma}+\frac{1}{2} \Omega_{+,-+}\right]\left(f+g_{2}-i g_{1}\right)=0 .
$$

Taking the sum and the difference of the above two equations, we deduce

$$
\partial_{+} f+\frac{i}{2}\left(g_{2}-i g_{1}\right) Q_{+}+\left[\frac{1}{2} \Omega_{+, \gamma}^{\gamma}+\frac{1}{2} \Omega_{+,-+}\right] f=0
$$

and

$$
\partial_{+}\left(g_{2}-i g_{1}\right)+\frac{i}{2} f Q_{+}+\left[\frac{1}{2} \Omega_{+, \gamma}^{\gamma}+\frac{1}{2} \Omega_{+,-+}\right]\left(g_{2}-i g_{1}\right)=0
$$

Taking the complex conjugate of (B.10), we find that

$$
\frac{i g_{2}}{f} Q_{+}+\Omega_{+, \gamma}^{\gamma}=0
$$

and

$$
\partial_{+} f+\frac{g_{1}}{2} Q_{+}+\frac{1}{2} \Omega_{+,-+} f=0
$$

Then (B.11) implies that

$$
-\frac{1}{2 f}\left(f^{2}-g_{2}^{2}\right) Q_{+}+\partial_{+} g_{1}+\frac{1}{2} \Omega_{+,-+} g_{1}=0
$$




$$
-\frac{g_{1} g_{2}}{2 f} Q_{+}+\partial_{+} g_{2}+\frac{1}{2} \Omega_{+,-+} g_{2}=0
$$

Next let us turn to (3.10) and (3.16) to determine $G_{+\alpha \bar{\beta}}$. Solving the latter in terms of $G_{+\alpha \bar{\beta}}$, we find

$$
-\frac{1}{2} G_{+\alpha \bar{\beta}}=\frac{f+g_{2}+i g_{1}}{f-g_{2}-i g_{1}}\left[\Omega_{\bar{\beta},+\alpha}-i F_{+\alpha \bar{\beta} \delta}{ }^{\delta}\right] .
$$

Next substituting $G_{+\alpha \bar{\beta}}$ in (3.10), and taking its complex conjugate, we find that

$$
\Omega_{\alpha, \bar{\beta}+}+\Omega_{\bar{\beta}, \alpha+}=0
$$

and

$$
i\left(f^{2}+g_{2}^{2}+g_{1}^{2}\right) F_{+\alpha \bar{\beta} \delta}{ }^{\delta}+2 f g_{2} \Omega_{\alpha,+\bar{\beta}}=0 .
$$

This equation determines the $F_{+\alpha \bar{\beta} \delta} \delta$ component of the flux $F$ in terms of the geometry. Substituting (B.17) in (B.15), we can determine $G_{+\alpha \bar{\beta}}$ in terms of the geometry as

$$
G_{+\alpha \bar{\beta}}=2 \frac{\left(f+i g_{1}\right)^{2}-g_{2}^{2}}{f^{2}+g_{2}^{2}+g_{1}^{2}} \Omega_{\alpha,+\bar{\beta}} .
$$

Next we turn our attention to (3.11), (3.15) and (3.6). First, we symmetrize the free indices in (3.15) and in the dual of (3.11) to find

$$
F_{+\bar{\beta}_{1} \bar{\beta}_{2} \bar{\beta}_{3}(\alpha} \epsilon_{\gamma)} \bar{\beta}_{1} \bar{\beta}_{2} \bar{\beta}_{3}=0
$$

which together with the duality constraint implies

$$
F_{+\alpha_{1} \alpha_{2} \alpha_{3} \bar{\beta}}=0
$$

and

$$
\Omega_{\alpha,+\beta}+\Omega_{\beta,+\alpha}=0
$$

Next, we dualize (3.11) and take the difference with the dual of (3.15) in such a way as to eliminate the $G$ dependence and to find

$$
\left(f+g_{2}+i g_{1}\right) \Omega_{\alpha,+\beta}-\frac{1}{2}\left(f-g_{2}+i g_{1}\right) \epsilon_{\alpha \beta} \bar{\gamma}_{1} \bar{\gamma}_{2} \Omega_{\bar{\gamma}_{1},+\bar{\gamma}_{2}}=0 .
$$

Comparing this expression with its complex conjugate, we get that

$$
\Omega_{\alpha,+\beta}=0
$$

Taking the sum and the difference of (3.6) and (3.15) and comparing the two, we deduce that

$$
G_{+\bar{\alpha} \bar{\beta}}=0
$$


and

$$
G_{\alpha \beta+}=0
$$

To continue, we substitute (B.24) and (B.25) into (3.23) and then use (B.23). After some computation, we find that

$$
\Omega_{+, \bar{\alpha} \bar{\beta}}=0 .
$$

This relates the $\Omega_{+, \bar{\alpha} \bar{\beta}}$ component of the connection to the $\Omega_{\gamma_{1},+\gamma_{2}}$ component.

Next, we multiply (3.17) with $f-g_{2}-i g_{1}$ and (3.19) with $f+g_{2}-i g_{1}$ and take their sum. Separating the resulting expression in real and imaginary parts, we get

$$
\partial_{-}\left(f^{2}+g_{2}^{2}+g_{1}^{2}\right)+\Omega_{-,-+}\left(f^{2}+g_{2}^{2}+g_{1}^{2}\right)=0
$$

and

$$
\begin{aligned}
& -i\left(f^{2}+g_{1}^{2}+g_{2}^{2}\right) Q_{-}+2 i f \partial_{-} g_{1}-2 i g_{1} \partial_{-} f-2 f g_{2} \Omega_{-, \alpha}{ }^{\alpha}+\frac{i}{2} F_{-\alpha}{ }^{\alpha}{ }^{\beta}\left(f^{2}+g_{2}^{2}+g_{1}^{2}\right) \\
& +\frac{i}{12}\left[f^{2}-\left(g_{2}+i g_{1}\right)^{2}\right] F_{-\gamma_{1} \gamma_{2} \gamma_{3} \gamma_{4}} \epsilon^{\gamma_{1} \gamma_{2} \gamma_{3} \gamma_{4}}
\end{aligned}
$$

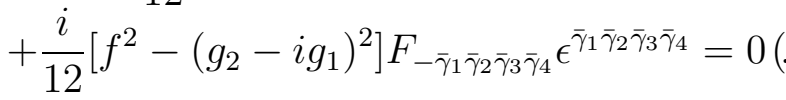

This equation can be thought of as determining $F_{-\alpha}{ }^{\alpha}{ }_{\beta}{ }^{\beta}$ in terms of the other fluxes and

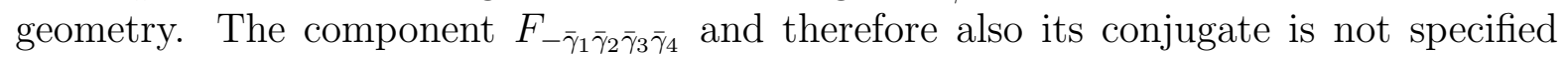
by the Killing spinor equations. We multiply (3.17) with $f+g_{2}+i g_{1}$ and (3.19) with $f-g_{2}+i g_{1}$ and take the difference. This gives

$$
\begin{aligned}
& -2 f \partial_{-} g_{2}+2 g_{2} \partial_{-} f+2 i g_{2} \partial_{-} g_{1}-2 i g_{1} \partial_{-} g_{2}+\Omega_{-, \alpha}{ }^{\alpha}\left[\left(f+i g_{1}\right)^{2}-g_{2}^{2}\right] \\
& +\frac{1}{2}\left(f^{2}+g_{2}^{2}+g_{1}^{2}\right) G_{-\alpha}{ }^{\alpha}+\frac{i}{12}\left(f+g_{2}+i g_{1}\right)^{2} F_{-\gamma_{1} \gamma_{2} \gamma_{3} \gamma_{4}} \epsilon^{\gamma_{1} \gamma_{2} \gamma_{3} \gamma_{4}}
\end{aligned}
$$

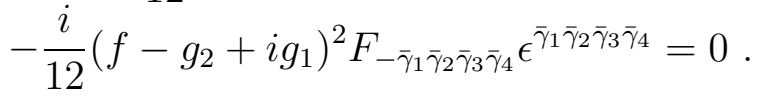

This equation expresses the component $G_{-\alpha}{ }^{\alpha}$ of the $G$ flux in terms of the $F$ flux and geometry.

The equation (3.18) contains the components $F_{-\alpha \beta \delta} \delta, G_{-\alpha \beta}$ and $G_{-\bar{\alpha} \bar{\beta}}$. So it can be used to determine either $G_{-\alpha \beta}$ or $G_{-\bar{\alpha} \bar{\beta}}$ in terms of the other two components. There is no obvious advantage to give explicitly the solution since the remaining fluxes are not determined by the rest of the equations of the linear system.

The conditions (3.8), (3.12), (3.14) and (3.20) should be investigated together. First, we take the trace of (3.8) to find

$$
\begin{array}{r}
\left(f-g_{2}+i g_{1}\right)\left[\Omega_{\beta}{ }^{\beta}{ }_{\bar{\alpha}}-i F_{-+\beta}{ }^{\beta} \bar{\alpha}\right]+\left(f+g_{2}-i g_{1}\right)\left[\frac{1}{8} G_{\bar{\alpha} \beta}{ }^{\beta}-\frac{3}{8} G_{\bar{\alpha}-+}\right] \\
-\frac{1}{2}\left(f+g_{2}+i g_{1}\right) \Omega_{\gamma_{1}, \gamma_{2} \gamma_{3}} \epsilon_{\bar{\alpha}}^{\gamma_{1} \gamma_{2} \gamma_{3}}-\frac{1}{8}\left(f-g_{2}-i g_{1}\right) G_{\gamma_{1} \gamma_{2} \gamma_{3}} \epsilon^{\gamma_{1} \gamma_{2} \gamma_{3}}{ }_{\bar{\alpha}}=0 .
\end{array}
$$

Combining the above equation with (3.20), we get

$$
\left(f-g_{2}+i g_{1}\right)\left[\Omega_{\beta}{ }^{\beta}{ }_{\bar{\alpha}}+\Omega_{-,+\bar{\alpha}}\right]-\left(f+g_{2}+i g_{1}\right)\left[\frac{1}{2} \Omega_{\gamma_{1}, \gamma_{2} \gamma_{3}}+\frac{i}{3} F_{\gamma_{1} \gamma_{2} \gamma_{3}-+}\right] \epsilon^{\gamma_{1} \gamma_{2} \gamma_{3}} \bar{\alpha}
$$




$$
-\frac{1}{12}\left(f-g_{2}-i g_{1}\right) G_{\gamma_{1} \gamma_{2} \gamma_{3}} \epsilon^{\gamma_{1} \gamma_{2} \gamma_{3}} \bar{\alpha}=0
$$

which determines $G_{\gamma_{1} \gamma_{2} \gamma_{3}}$ in terms of the $F$ flux and geometry. Substituting the above solution for $G_{\gamma_{1} \gamma_{2} \gamma_{3}}$ in both (3.20) and (3.12), and summing them with appropriate numerical factors, we get

$$
\begin{aligned}
& D_{\bar{\alpha}}\left(f-g_{2}+i g_{1}\right)+\left[\frac{1}{2} \Omega_{\bar{\alpha}, \beta}{ }^{\beta}+\frac{1}{2} \Omega_{\bar{\alpha},-+}+\Omega_{\beta}{ }^{\beta}{ }_{\bar{\alpha}}+i F_{-+\bar{\alpha} \beta}{ }^{\beta}+2 \Omega_{-,+\bar{\alpha}}\right]\left(f-g_{2}+i g_{1}\right) \\
& \quad+\left(f+g_{2}+i g_{1}\right)\left[-\frac{1}{2} \Omega_{\gamma_{1}, \gamma_{2} \gamma_{3}}-\frac{i}{3} F_{\gamma_{1} \gamma_{2} \gamma_{3}-+}\right] \epsilon^{\gamma_{1} \gamma_{2} \gamma_{3}}{ }_{\bar{\alpha}}+\frac{1}{2} G_{-+\bar{\alpha}}\left(f+g_{2}-i g_{1}\right)=0
\end{aligned}
$$

This equation determines $G_{-+\bar{\alpha}}$ in terms of the $F$ fluxes and the geometry. If instead, we take the difference of (3.12) and (B.32) with appropriate numerical factors, we get

$$
\begin{array}{r}
\frac{3}{4} D_{\bar{\alpha}}\left(f-g_{2}+i g_{1}\right)+\left[\frac{3}{8} \Omega_{\bar{\alpha}, \beta}{ }^{\beta}+\frac{3}{8} \Omega_{\bar{\alpha},-+}\right. \\
\left.-\frac{i}{4} F_{-+\bar{\alpha} \beta}{ }^{\beta}+\frac{1}{4} \Omega_{\beta}{ }^{\beta}{ }_{\bar{\alpha}}\right]\left(f-g_{2}+i g_{1}\right)+\frac{1}{8} G_{\bar{\alpha} \beta}^{\beta}\left(f+g_{2}-i g_{1}\right) \\
+\left(f+g_{2}+i g_{1}\right)\left[-\frac{1}{8} \Omega_{\gamma_{1}, \gamma_{2} \gamma_{3}}+\frac{i}{4} F_{\gamma_{1} \gamma_{2} \gamma_{3}-+}\right] \epsilon^{\gamma_{1} \gamma_{2} \gamma_{3}} \bar{\alpha}=0
\end{array}
$$

which determines the component $G_{\bar{\alpha} \beta}{ }^{\beta}$ of $G$. By substituting the above results into (3.8), we can solve for $G_{\alpha \bar{\beta} \bar{\gamma}}$

$$
\begin{array}{r}
G_{\alpha \bar{\beta} \bar{\gamma}}=\frac{1}{\left(f+g_{2}-i g_{1}\right)}\left(-4 g_{\alpha[\bar{\beta}} D_{\bar{\gamma}]}\left(f-g_{2}+i g_{1}\right)+\left(f-g_{2}+i g_{1}\right)\left(-2 \Omega_{\alpha, \bar{\beta} \bar{\gamma}}\right.\right. \\
\left.-2 g_{\alpha[\bar{\beta}} \Omega_{\bar{\gamma}], \delta} \delta+2 g_{\alpha[\bar{\beta}} \Omega_{\bar{\gamma}],+-}-4 i F_{-+\alpha \bar{\beta} \bar{\gamma}}+4 i g_{\alpha[\bar{\beta}} F_{\bar{\gamma}]-+\delta}\right) \\
\left.+\left(f+g_{2}+i g_{1}\right) \epsilon_{\bar{\beta} \bar{\gamma}} \delta_{1} \delta_{2}\left(\Omega_{\alpha, \delta_{1} \delta_{2}}-2 i F_{-+\alpha \delta_{1} \delta_{2}}\right)\right) .
\end{array}
$$

We have thus solved all three equations (3.8), (3.12), and (3.20) for $G_{\alpha \beta \gamma}, G_{-+\bar{\alpha}}, G_{\bar{\alpha} \beta}{ }^{\beta}$ and $G_{\alpha \bar{\beta} \bar{\gamma}}$.

It remains to solve (3.14). For this first, we compute the difference (B.33) and (B.32) with appropriate numerical factors and find

$$
\begin{array}{r}
D_{\bar{\alpha}}\left(f-g_{2}+i g_{1}\right)+\left[\frac{1}{2} \Omega_{\bar{\alpha}, \beta} \beta+\frac{1}{2} \Omega_{\bar{\alpha},-+}-i F_{-+\bar{\alpha} \beta} \beta\right. \\
\left.-\Omega_{-,+\bar{\alpha}}\right]\left(f-g_{2}+i g_{1}\right)+\left[-\frac{1}{4} G_{-+\bar{\alpha}}+\frac{1}{4} G_{\bar{\alpha} \beta}^{\beta}\right]\left(f+g_{2}-i g_{1}\right) \\
+\frac{2 i}{3}\left(f+g_{2}+i g_{1}\right) F_{\gamma_{1} \gamma_{2} \gamma_{3}-+} \epsilon^{\gamma_{1} \gamma_{2} \gamma_{3}}{ }_{\bar{\alpha}}=0 .
\end{array}
$$

Next we multiply (B.35) with $f-g_{2}-i g_{1}$ and (3.14) with $f+g_{2}-i g_{1}$ to get

$$
\begin{array}{r}
\partial_{\bar{\alpha}}\left(f^{2}+g_{2}^{2}+g_{1}^{2}\right)-i Q_{\bar{\alpha}}\left(f^{2}+g_{2}^{2}+g_{1}^{2}\right)+2 i f D_{\bar{\alpha}} g_{1}-2 i g_{1} D_{\bar{\alpha}} f+\left[\Omega_{\bar{\alpha},-+}{ }_{\beta}-\Omega_{-,+\bar{\alpha}}\right] \\
\left.-2 i F_{-+\bar{\alpha} \beta}{ }^{\beta}-\Omega_{-,+\bar{\alpha}}\right]\left(f^{2}+g_{2}^{2}+g_{1}^{2}\right)-2 f g_{2}\left[\Omega_{\bar{\alpha}, \beta}{ }^{2}-\Omega_{1}\right) \\
+\frac{2 i}{3}\left(f^{2}-\left(g_{2}+i g_{1}\right)^{2}\right) F_{\gamma_{1} \gamma_{2} \gamma_{3}-+} \epsilon^{\gamma_{1} \gamma_{2} \gamma_{3}}{ }_{\bar{\alpha}}=0 .
\end{array}
$$

This will be compared later with another equation which we shall derive by examining the rest of the equations. 
Similarly, the conditions (3.7), (3.9), (3.13) and (3.21) should be investigated together. We first dualize (3.13) and then take the trace to find

$$
\begin{aligned}
& \left(f+g_{2}+i g_{1}\right)\left[-\Omega_{\bar{\beta},{ }^{\bar{\beta}} \alpha}-i F_{-+\alpha \beta}{ }^{\beta}\right]+\left(f-g_{2}-i g_{1}\right)\left[\frac{1}{8} G_{\alpha \beta}{ }^{\beta}+\frac{3}{8} G_{\alpha-+}\right] \\
+ & \frac{1}{2}\left(f-g_{2}+i g_{1}\right) \Omega_{\bar{\gamma}_{1}, \bar{\gamma}_{2} \bar{\gamma}_{3}} \epsilon^{\bar{\gamma}_{1} \bar{\gamma}_{2} \bar{\gamma}_{3}}{ }_{\alpha}+\frac{1}{8}\left(f+g_{2}-i g_{1}\right) G_{\bar{\gamma}_{1} \bar{\gamma}_{2} \bar{\gamma}_{3}} \epsilon^{\bar{\gamma}_{1} \bar{\gamma}_{2} \bar{\gamma}_{3}}{ }_{\alpha}=0 .
\end{aligned}
$$

We take the sum of (B.37) with the dual of (3.21) after weighting the equations with appropriate coefficients to get

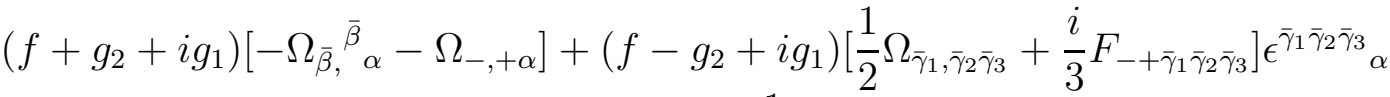

$$
\begin{aligned}
& +\frac{1}{12}\left(f+g_{2}-i g_{1}\right) G_{\bar{\gamma}_{1} \bar{\gamma}_{2} \bar{\gamma}_{3}} \epsilon_{\alpha}^{\bar{\gamma}_{1} \bar{\gamma}_{2} \bar{\gamma}_{3}}=0 \text {. }
\end{aligned}
$$

This equation gives $G_{\bar{\gamma}_{1} \bar{\gamma}_{2} \bar{\gamma}_{3}}$ in terms of the $F$ flux and the geometry.

We substitute $G_{\bar{\gamma}_{1} \bar{\gamma}_{2} \bar{\gamma}_{3}}$ in the dual of (13.21) and in (3.9) and take their difference after weighting the equations with appropriate coefficients to find

$$
\begin{aligned}
& D_{\alpha}\left(f+g_{2}+i g_{1}\right)+\left[-\frac{1}{2} \Omega_{\alpha, \beta}{ }^{\beta}+\frac{1}{2} \Omega_{\alpha,-+}-i F_{-+\alpha \gamma}{ }^{\gamma}\right.
\end{aligned}
$$

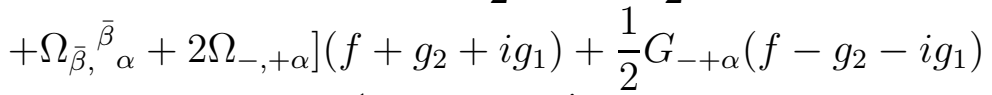

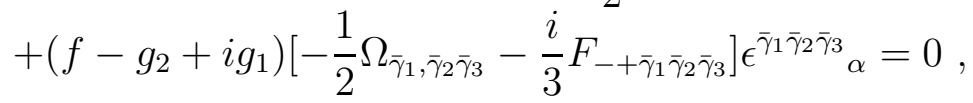

which gives $G_{-+\alpha}$ in terms of the other fluxes and geometry. By substituting the above results into (3.13), we can now solve for $G_{\alpha \beta \bar{\gamma}}$ to get

$$
\begin{array}{r}
G_{\alpha \beta \bar{\gamma}}=\frac{1}{\left(f-g_{2}-i g_{1}\right)}\left(-4 g_{\bar{\gamma}[\alpha} D_{\beta]}\left(f+g_{2}+i g_{1}\right)+\left(f+g_{2}+i g_{1}\right)\left(-2 \Omega_{\bar{\gamma}, \alpha \beta}\right.\right. \\
\left.+2 g_{\bar{\gamma}[\alpha} \Omega_{\beta], \delta}{ }^{\delta}+2 g_{\bar{\gamma}[\alpha} \Omega_{\beta],+-}-4 i F_{-+\alpha \beta \bar{\gamma}}-4 i g_{\bar{\gamma}[\alpha} F_{\beta]-+\delta}\right) \\
\left.+\left(f-g_{2}+i g_{1}\right) \epsilon_{\alpha \beta} \bar{\delta}_{1} \bar{\delta}_{2}\left(\Omega_{\bar{\gamma}, \bar{\delta}_{1} \bar{\delta}_{2}}-2 i F_{-+\bar{\gamma} \bar{\delta}_{1} \bar{\delta}_{2}}\right)\right) .
\end{array}
$$

Subtracting (B.39) from (3.9) with appropriate factors, we find

$$
\begin{aligned}
& \frac{3}{4} D_{\alpha}\left(f+g_{2}+i g_{1}\right)+\left[-\frac{3}{8} \Omega_{\alpha, \beta} \beta+\frac{3}{8} \Omega_{\alpha,-+}+\frac{i}{4} F_{-+\alpha \delta} \delta\right. \\
& \left.+\frac{1}{4} \Omega_{\bar{\beta}}{ }^{\bar{\beta}}{ }_{\alpha}\right]\left(f+g_{2}+i g_{1}\right)-\frac{1}{8} G_{\alpha \delta} \delta\left(f-g_{2}-i g_{1}\right)
\end{aligned}
$$

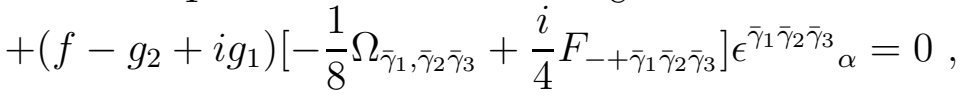

which gives $G_{\alpha \delta} \delta$ in terms of the $F$ flux and geometry.

Subtracting (B.39) from (B.41) with appropriate numerical factors, we find

$$
\begin{array}{r}
D_{\alpha}\left(f+g_{2}+i g_{1}\right)+\left[-\frac{1}{2} \Omega_{\alpha, \beta}{ }^{\beta}+\frac{1}{2} \Omega_{\alpha,-+}+i F_{-+\alpha \delta} \delta-\Omega_{-,+\alpha}\right]\left(f+g_{2}+i g_{1}\right) \\
+\left[-\frac{1}{4} G_{\alpha \gamma}{ }^{\gamma}-\frac{1}{4} G_{-+\alpha}\right]\left(f-g_{2}-i g_{1}\right)+\frac{2 i}{3}\left(f-g_{2}+i g_{1}\right) F_{-+\bar{\gamma}_{1} \bar{\gamma}_{2} \bar{\gamma}_{3}} \epsilon^{\bar{\gamma}_{1} \bar{\gamma}_{2} \bar{\gamma}_{3}}{ }_{\alpha}=0 .
\end{array}
$$


We multiply (3.7) with $f-g_{2}-i g_{1}$ and (B.42) with $f+g_{2}-i g_{1}$ and sum them together. Then we take the complex conjugate of the resulting expression to find

$$
\begin{array}{r}
\partial_{\bar{\alpha}}\left(f^{2}+g_{2}^{2}+g_{1}^{2}\right)+i Q_{\bar{\alpha}}\left(f^{2}+g_{2}^{2}+g_{1}^{2}\right)-2 i f \bar{D}_{\bar{\alpha}} g_{1}+2 i g_{1} \bar{D}_{\bar{\alpha}} f \\
+\left[\Omega_{\bar{\alpha},-+}+2 i F_{-+\bar{\alpha} \beta}{ }^{\beta}-\Omega_{-,+\bar{\alpha}}\right]\left(f^{2}+g_{2}^{2}+g_{1}^{2}\right)-2 f g_{2}\left[-\Omega_{\bar{\alpha}, \beta}{ }^{\beta}+\Omega_{-,+\bar{\alpha}}\right] \\
-\frac{2 i}{3}\left(f^{2}-\left(g_{2}+i g_{1}\right)^{2}\right) F_{-+\gamma_{1} \gamma_{2} \gamma_{3}} \epsilon^{\gamma_{1} \gamma_{2} \gamma_{3}}{ }_{\bar{\alpha}}=0 .
\end{array}
$$

It remains to compare (B.36) with (B.43). Taking the sum and the difference, we find

$$
\partial_{\bar{\alpha}}\left(f^{2}+g_{2}^{2}+g_{1}^{2}\right)-\left[\Omega_{\bar{\alpha},+-}+\Omega_{-,+\bar{\alpha}}\right]\left(f^{2}+g_{2}^{2}+g_{1}^{2}\right)=0
$$

and

$$
\begin{aligned}
- & 2 i\left(f^{2}+g_{2}^{2}+g_{1}^{2}\right) Q_{\bar{\alpha}}+4 i f \partial_{\bar{\alpha}} g_{1}-4 i g_{1} \partial_{\bar{\alpha}} f-4 i F_{-+\bar{\alpha} \beta}{ }^{\beta}\left(f^{2}+g_{2}^{2}+g_{1}^{2}\right) \\
- & 4 f g_{2}\left[\Omega_{\bar{\alpha}, \beta}{ }^{\beta}-\Omega_{-,+\bar{\alpha}}\right]+\frac{4 i}{3}\left(f^{2}-\left(g_{2}+i g_{1}\right)^{2}\right) F_{-+\gamma_{1} \gamma_{2} \gamma_{3}} \epsilon^{\gamma_{1} \gamma_{2} \gamma_{3}} \bar{\alpha}=0 .
\end{aligned}
$$

These last equation can be used to determine one more component of the $F$ flux, say $F_{-+\gamma_{1} \gamma_{2} \gamma_{3}}$. We shall not substitute $F_{-+\gamma_{1} \gamma_{2} \gamma_{3}}$ back into the equations that determine the $G$ fluxes. This is because the resulting equations do not exhibit any apparent simplification. So, we shall take the scalar fluxes to depend on the $G$ and so implicitly on the $F$ fluxes and geometry, the $G$ fluxes to depend on the $F$ fluxes and geometry, and the $F$ fluxes to depend on the geometry. The equations that determine the various components of the fluxes are summarized in the tables below.

The scalar fluxes $P$ are given in the following equations

\begin{tabular}{|c|c|}
\hline Fluxes & Equations \\
\hline$P_{+}$ & $(\mathrm{B} .6)$ \\
$P_{\alpha}$ & $(3.3)$ \\
$P_{\bar{\alpha}}$ & $(3.2)$ \\
\hline
\end{tabular}

The $G$ fluxes are determined by the following equations

\begin{tabular}{|c|c|}
\hline Fluxes & Equations \\
\hline$G_{+\alpha}{ }^{\alpha}$ & $(\mathrm{B} .6)$ \\
$G_{+\alpha \bar{\beta}}$ & $(\mathrm{B} .18$ \\
$G_{+\alpha \beta}$ & $(\mathrm{B} .25$ \\
$G_{+\bar{\alpha} \bar{\beta}}$ & $(\mathrm{B} .24$ \\
$G_{-\alpha}{ }^{2}$ & $(\mathrm{~B} .29)$ \\
$G_{-\alpha \beta}$ & $(\mathrm{B} .18)$ \\
$G_{\alpha \beta \gamma}$ & $(\mathrm{B} .31$ \\
$G_{-+\bar{\alpha}}$ & $(\mathrm{B} .32$ \\
$G_{\alpha \bar{\beta} \bar{\gamma}}$ & $(\mathrm{B} .34$ \\
$G_{\bar{\alpha} \bar{\beta} \bar{\gamma}}$ & $(\mathrm{B} .38$ \\
$G_{-+\alpha}$ & $(\mathrm{B} .39$ \\
$G_{\alpha \beta \bar{\gamma}}$ & $(\mathrm{B} .40$ \\
\hline
\end{tabular}


The $F$ fluxes are determined by the following equations

\begin{tabular}{|l|c|}
\hline Fluxes & Equations \\
\hline$F_{+\alpha \beta \gamma \delta}$ & $(\mathrm{B} .4)$ \\
$F_{+\alpha \bar{\beta} \gamma}{ }^{\gamma}$ & $(\mathrm{B} .17$ \\
$F_{+\alpha \beta \gamma \delta}$ & $(\mathrm{B} .20)$ \\
$F_{-\alpha}{ }^{\alpha} \beta$ & $(\mathrm{B} .28$ \\
$F_{-+\alpha \beta \gamma}$ & $(\mathrm{B} .45$ \\
\hline
\end{tabular}

The fluxes that are not mentioned in the above tables are not restricted by the Killing spinor equations. $F$ is further restricted by the self-duality condition. The conditions on the geometry have been summarized in section three.

\section{Appendix C The solution of Killing spinor equa- tions for the $\operatorname{Spin}(7) \ltimes \mathbb{R}^{8}$-invariant spinor}

The analysis of the conditions of the Killing spinor equations for a $\operatorname{Spin}(7) \ltimes \mathbb{R}^{8}$ invariant spinor is similar to that of an $S U(4) \ltimes \mathbb{R}^{8}$ invariant spinor but there are some differences. Because of this and for stating the conditions for the existence of a parallel $\operatorname{Spin}(7) \ltimes \mathbb{R}^{8}$ spinor, we shall repeat the analysis from the beginning.

The algebraic Killing spinor equations (3.4) and (3.5) imply

$$
P_{+}=G_{+\alpha}{ }^{\alpha}=0,
$$

and (3.6) gives

$$
G_{+\bar{\alpha} \bar{\beta}}-\frac{1}{2} \epsilon_{\bar{\alpha} \bar{\beta}}{ }^{\gamma \delta} G_{+\gamma \delta}=0
$$

The two other equations can be thought of as determining the scalars in terms of the $G$ fluxes.

Next consider (3.16) and (3.10). After taking the dual of the former and the trace, and using the self-duality of $F$, we find

$$
\Omega_{\alpha,+}{ }^{\alpha}=0
$$

Using the above equations, we take the sum and the difference of (3.10) with the dual of (3.16), and separate the sum in real and imaginary parts to find

$$
\Omega_{\alpha,+\bar{\beta}}+\Omega_{\bar{\beta},+\alpha}=0
$$

and

$$
F_{+\alpha \bar{\beta} \delta}^{\delta}=0
$$


The difference, instead, gives

$$
(f-i g) G_{+\alpha \bar{\beta}}-2(f+i g) \Omega_{\alpha,+\bar{\beta}}=0 .
$$

Using (3.22) and (3.24) and some of the above equations, we find that

$$
\Omega_{+, \gamma}^{\gamma}=0,
$$

and

$$
\begin{aligned}
g Q_{+}+2 \partial_{+} f+\Omega_{+,-+} f & =0, \\
-f Q_{+}+2 \partial_{+} g+\Omega_{+,-+} g & =0 .
\end{aligned}
$$

We now consider (3.6), (3.11) and (3.15). Symmetrizing (3.11) and (3.15) and comparing them, we get

$$
\Omega_{(\alpha, \beta)+}=0, \quad F_{+\bar{\gamma}_{1} \bar{\gamma}_{2} \bar{\gamma}_{3}(\alpha} \epsilon_{\beta)} \bar{\gamma}_{1} \bar{\gamma}_{2} \bar{\gamma}_{3}=0 .
$$

The latter condition together with the self-duality of $F$ imply

$$
F_{\alpha_{1} \alpha_{2} \alpha_{3} \bar{\beta}}=0 .
$$

Therefore the symmetric part of (3.15) and of the dual of (3.11) vanishes identically. Next, we take the difference of (3.11) with (3.15) with appropriate factors to find

$$
\Omega_{\alpha,+\beta}-\frac{1}{2} \epsilon_{\alpha \beta} \bar{\gamma}_{1} \bar{\gamma}_{2} \Omega_{\bar{\gamma}_{1},+\bar{\gamma}_{2}}=0 .
$$

The conditions (3.6), (3.11) and (3.15) give

$$
G_{+\alpha \beta}-2 \frac{f+i g}{f-i g} \Omega_{\alpha,+\beta}=0,
$$

and

$$
G_{+\bar{\alpha} \bar{\beta}}-2 \frac{f+i g}{f-i g} \Omega_{\bar{\alpha},+\bar{\beta}}=0,
$$

which determine the components of the $G$ flux in terms of the geometry.

Next let us turn our attention to (3.23). Using (C.12) and (C.13), we find that

$$
\Omega_{+, \bar{\alpha} \bar{\beta}}-\frac{1}{2} \epsilon_{\bar{\alpha} \bar{\beta}} \gamma_{1} \gamma_{2} \Omega_{+, \bar{\gamma}_{1} \bar{\gamma}_{2}}=0 .
$$

Taking the difference of (3.17) and (3.19), we get

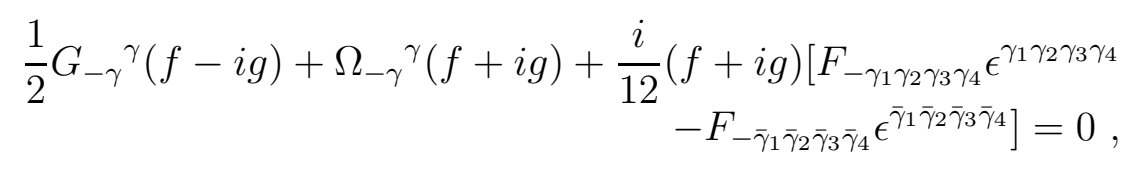


which can be used to determine $G_{-\gamma}$. Then splitting the sum of (3.17) and (3.19) in real and imaginary pieces, we find

$$
\begin{aligned}
& 2 \partial_{-} f+Q_{-} g+\Omega_{-,-+} f-\frac{1}{2} F_{-\gamma}{ }^{\gamma} \delta g-\frac{1}{12} g\left[F_{-\gamma_{1} \gamma_{2} \gamma_{3} \gamma_{4}} \epsilon^{\gamma_{1} \gamma_{2} \gamma_{3} \gamma_{4}}\right.
\end{aligned}
$$

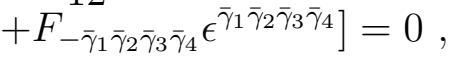

and

$$
\begin{aligned}
& 2 \partial_{-} g-Q_{-} f+\Omega_{-,-+} g+\frac{f}{2} F_{-\gamma}{ }^{\gamma} \delta+\frac{f}{12}\left[F_{-\gamma_{1} \gamma_{2} \gamma_{3} \gamma_{4}} \epsilon^{\gamma_{1} \gamma_{2} \gamma_{3} \gamma_{4}}\right.
\end{aligned}
$$

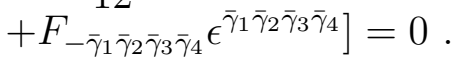

The last two equations give

$$
\partial_{-}\left(f^{2}+g^{2}\right)+\Omega_{-,-+}\left(f^{2}+g^{2}\right)=0,
$$

and

$$
\begin{array}{r}
2 g \partial_{-} f-2 f \partial_{-} g+Q_{-}\left(f^{2}+g^{2}\right)-\frac{1}{2} F_{-\gamma^{\gamma} \delta}{ }^{\delta}\left(g^{2}+f^{2}\right) \\
-\frac{f^{2}+g^{2}}{12}\left[F_{-\gamma_{1} \gamma_{2} \gamma_{3} \gamma_{4}} \epsilon^{\gamma_{1} \gamma_{2} \gamma_{3} \gamma_{4}}+F_{-\bar{\gamma}_{1} \bar{\gamma}_{2} \bar{\gamma}_{3} \bar{\gamma}_{4}} \epsilon^{\bar{\gamma}_{1} \bar{\gamma}_{2} \bar{\gamma}_{3} \bar{\gamma}_{4}}\right]=0 .
\end{array}
$$

The latter can be used to determine $F_{-\gamma} \delta^{\delta}$.

The condition (3.18) can be written as

$$
\begin{array}{r}
{\left[\Omega_{-, \bar{\beta}_{1} \bar{\beta}_{2}}-\frac{1}{2} \epsilon_{\bar{\beta}_{1} \bar{\beta}_{2}}{ }^{\gamma_{1} \gamma_{2}} \Omega_{-, \gamma_{1} \gamma_{2}}\right.} \\
\left.+i\left(F_{-\bar{\beta}_{1} \bar{\beta}_{2} \delta}{ }^{\delta}+\frac{1}{2} \epsilon_{\bar{\beta}_{1} \bar{\beta}_{2}}^{\gamma_{1} \gamma_{2}} F_{-\gamma_{1} \gamma_{2} \delta} \delta\right)\right](f+i g) \\
+\frac{(f-i g)}{2}\left(G_{-\bar{\beta}_{1} \bar{\beta}_{2}}-\frac{1}{2} \epsilon_{\bar{\beta}_{1} \bar{\beta}_{2}}{ }^{\gamma_{1} \gamma_{2}} G_{-\gamma_{1} \gamma_{2}}\right)=0
\end{array}
$$

and can be used to determine the complex anti-self dual part of $G_{-\bar{\alpha} \bar{\beta}}$.

The equations (3.8), (3.12), (3.14) and (3.20) can be analyzed as in the case with an $S U(4) \ltimes \mathbb{R}^{8}$ invariant spinor. We shall not give the details but instead we shall state the results. In particular $G_{\alpha \beta \gamma}$ is determined by the equation

$$
\begin{aligned}
& (f+i g)\left[\Omega_{\beta}{ }^{\beta}{ }_{\bar{\alpha}}+\Omega_{-,+\bar{\alpha}}\right]-(f+i g)\left[\frac{1}{2} \Omega_{\gamma_{1}, \gamma_{2} \gamma_{3}}+\frac{i}{3} F_{\gamma_{1} \gamma_{2} \gamma_{3}-+}\right] \epsilon^{\gamma_{1} \gamma_{2} \gamma_{3}} \bar{\alpha} \\
& -\frac{1}{12}(f-i g) G_{\gamma_{1} \gamma_{2} \gamma_{3}} \epsilon_{\bar{\alpha}}^{\gamma_{1} \gamma_{2} \gamma_{3}}=0 \text {, }
\end{aligned}
$$

$G_{-+\bar{\alpha}}$ is determined by

$$
\begin{aligned}
& D_{\bar{\alpha}}(f+i g)+\left[\frac{1}{2} \Omega_{\bar{\alpha}, \beta}{ }^{\beta}+\frac{1}{2} \Omega_{\bar{\alpha},-+}+\Omega_{\beta,{ }^{\beta} \bar{\alpha}}+i F_{-+\bar{\alpha} \beta}{ }^{\beta}+2 \Omega_{-,+\bar{\alpha}}\right](f+i g) \\
& +(f+i g)\left[-\frac{1}{2} \Omega_{\gamma_{1}, \gamma_{2} \gamma_{3}}-\frac{i}{3} F_{\gamma_{1} \gamma_{2} \gamma_{3}-+}\right] \epsilon^{\gamma_{1} \gamma_{2} \gamma_{3}}+\frac{1}{2} G_{-+\bar{\alpha}}(f-i g)=0,
\end{aligned}
$$

and $G_{\bar{\alpha} \beta}{ }^{\beta}$ is determined by

$$
\frac{3}{4} D_{\bar{\alpha}}(f+i g)+\left[\frac{3}{8} \Omega_{\bar{\alpha}, \beta}{ }^{\beta}+\frac{3}{8} \Omega_{\bar{\alpha},-+}-\frac{i}{4} F_{-+\bar{\alpha} \beta}{ }^{\beta}+\frac{1}{4} \Omega_{\beta},_{\bar{\alpha}}\right](f+i g)
$$




$$
+(f+i g)\left[-\frac{1}{8} \Omega_{\gamma_{1}, \gamma_{2} \gamma_{3}}+\frac{i}{4} F_{\gamma_{1} \gamma_{2} \gamma_{3}-+}\right] \epsilon^{\gamma_{1} \gamma_{2} \gamma_{3}}{ }_{\bar{\alpha}}+\frac{1}{8} G_{\bar{\alpha} \beta}^{\beta}(f-i g)=0 .
$$

Substituting the above results into (3.8), we can solve for $G_{\alpha \bar{\beta} \bar{\gamma}}$ to get

$$
\begin{array}{r}
G_{\alpha \bar{\beta} \bar{\gamma}}=\frac{1}{(f-i g)}\left(-4 g_{\alpha[\bar{\beta}} D_{\bar{\gamma}]}(f+i g)+(f+i g)\left(-2 \Omega_{\alpha, \bar{\beta} \bar{\gamma}}\right.\right. \\
\left.-2 g_{\alpha[\bar{\beta}} \Omega_{\bar{\gamma}], \delta} \delta+2 g_{\alpha[\bar{\beta}} \Omega_{\bar{\gamma}],+-}-4 i F_{-+\alpha \bar{\beta} \bar{\gamma}}+4 i g_{\alpha[\bar{\beta}} F_{\bar{\gamma}]-+\delta}{ }^{\delta}\right) \\
\left.+(f+i g) \epsilon_{\bar{\beta} \bar{\gamma}}{ }^{\delta_{1} \delta_{2}}\left(\Omega_{\alpha, \delta_{1} \delta_{2}}-2 i F_{-+\alpha \delta_{1} \delta_{2}}\right)\right) .
\end{array}
$$

Similarly for the equations (3.7), (3.9), (3.13) and (3.21), we find that $G_{\bar{\alpha} \bar{\beta} \bar{\gamma}}$ is determined by

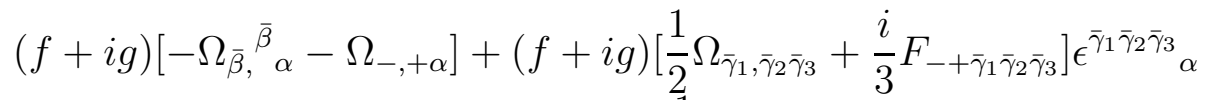

$$
\begin{aligned}
& +\frac{1}{12}(f-i g) G_{\bar{\gamma}_{1} \bar{\gamma}_{2} \bar{\gamma}_{3}} \epsilon^{\bar{\gamma}_{1} \bar{\gamma}_{2} \bar{\gamma}_{3}}{ }_{\alpha}=0 \text {, }
\end{aligned}
$$

$G_{-+\alpha}$ is determined by

$$
\begin{aligned}
& D_{\alpha}(f+i g)+\left[-\frac{1}{2} \Omega_{\alpha, \beta}{ }^{\beta}+\frac{1}{2} \Omega_{\alpha,-+}-i F_{-+\alpha \gamma}{ }^{\gamma}\right.
\end{aligned}
$$

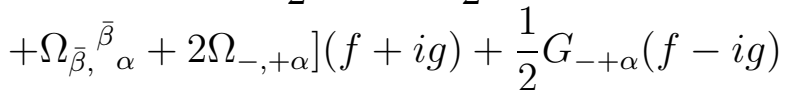

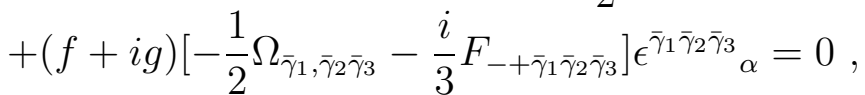

and $G_{\alpha \delta} \delta$ is determined by

$$
\begin{aligned}
& \frac{3}{4} D_{\alpha}(f+i g)+\left[-\frac{3}{8} \Omega_{\alpha, \beta}{ }^{\beta}+\frac{3}{8} \Omega_{\alpha,-+}+\frac{i}{4} F_{-+\alpha \delta} \delta+\frac{1}{4} \Omega_{\bar{\beta},}{ }^{\bar{\beta}}{ }_{\alpha}\right](f+i g) \\
& +(f+i g)\left[-\frac{1}{8} \Omega_{\bar{\gamma}_{1}, \bar{\gamma}_{2} \bar{\gamma}_{3}}+\frac{i}{4} F_{\left.-+\bar{\gamma}_{1} \bar{\gamma}_{2} \bar{\gamma}_{3}\right]} \epsilon_{\alpha}^{\bar{\gamma}_{1} \bar{\gamma}_{2} \bar{\gamma}_{3}}{ }_{\alpha}-\frac{1}{8} G_{\alpha \delta} \delta(f-i g)=0\right. \text {. }
\end{aligned}
$$

By substituting the above results into (3.13), we can solve for $G_{\alpha \beta \bar{\gamma}}$ to get

$$
\begin{aligned}
& G_{\alpha \beta \bar{\gamma}}=\frac{1}{(f-i g)}(-4 g_{\bar{\gamma}[\alpha} D_{\beta]}(f+i g)+(f+i g)\left(-2 \Omega_{\bar{\gamma}, \alpha \beta}\right. \\
&+2 g_{\bar{\gamma}[\alpha} \Omega_{\beta], \delta} \delta+\left.2 g_{\bar{\gamma}[\alpha} \Omega_{\beta],+-}-4 i F_{-+\alpha \beta \bar{\gamma}}-4 i g_{\bar{\gamma}[\alpha} F_{\beta]-+\delta} \delta\right) \\
&\left.+(f+i g) \epsilon_{\alpha \beta} \bar{\delta}_{1} \bar{\delta}_{2}\left(\Omega_{\bar{\gamma}, \bar{\delta}_{1} \bar{\delta}_{2}}-2 i F_{-+\bar{\gamma} \bar{\delta}_{1} \bar{\delta}_{2}}\right)\right)
\end{aligned}
$$

Using the above results, (3.7) and (3.14) yield

$$
\partial_{\bar{\alpha}}\left(f^{2}+g^{2}\right)-\left[\Omega_{\bar{\alpha},+-}+\Omega_{-,+\bar{\alpha}}\right]\left(f^{2}+g^{2}\right)=0,
$$

and

$$
\begin{array}{r}
4 i f \partial_{\bar{\alpha}} g-4 i g \partial_{\bar{\alpha}} f+\left[-2 i Q_{\bar{\alpha}}-4 i F_{-+\bar{\alpha} \beta}{ }^{\beta}\right]\left(f^{2}+g^{2}\right) \\
+\frac{4 i}{3}\left(f^{2}+g^{2}\right) F_{-+\gamma_{1} \gamma_{2} \gamma_{3}} \epsilon^{\gamma_{1} \gamma_{2} \gamma_{3}}=0 .
\end{array}
$$


The last equation can be used to determine $F_{-+\gamma_{1} \gamma_{2} \gamma_{3}}$. We summarize below the equations that give the components of the fluxes as in the $S U(4) \ltimes \mathbb{R}^{8}$ case.

The scalar fluxes $P$ are given in the following equations

\begin{tabular}{|c|c|}
\hline Fluxes & Equations \\
\hline$P_{+}$ & $(\overline{\text { C.1 }})$ \\
$P_{\alpha}$ & $(3.3)$ \\
$P_{\bar{\alpha}}$ & $(3.2)$ \\
\hline
\end{tabular}

The $G$ fluxes are determined by the following equations

\begin{tabular}{|c|c|}
\hline Fluxes & Equations \\
\hline$G_{+\alpha}{ }^{\alpha}$ & (C.1) \\
\hline$G_{+\alpha \bar{\beta}}$ & (C.6) \\
\hline$G_{+\alpha \beta}$ & (C.12) \\
\hline$G_{+\bar{\alpha} \bar{\beta}}$ & (C.13) \\
\hline$G_{-\alpha}^{\alpha}$ & (C.15) \\
\hline$G_{-\bar{\alpha} \bar{\beta}}$ & (C.20) \\
\hline$G_{\alpha \beta \gamma}$ & (C.21) \\
\hline$G_{-+\bar{\alpha}}$ & (C.22) \\
\hline$G_{\alpha \bar{\beta} \bar{\gamma}}$ & (C.24) \\
\hline$G_{\bar{\alpha} \bar{\beta} \bar{\gamma}}$ & (C.25) \\
\hline$G_{-+\alpha}$ & (C.26) \\
\hline$G_{\alpha \beta \bar{\gamma}}$ & (C.28) \\
\hline
\end{tabular}

The $F$ fluxes are determined by the following equations

\begin{tabular}{|c|c|}
\hline Fluxes & Equations \\
\hline$F_{+\alpha \beta \gamma \delta}$ & $(\mathbb{B} .4)$ \\
$F_{+\alpha \bar{\beta} \gamma} \gamma$ & $(\mathrm{C} .5)$ \\
$F_{+\alpha \beta \gamma \bar{\delta}}$ & $(\mathrm{C} .10$ \\
$F_{-\alpha}{ }_{\beta} \beta$ & $(\mathbf{C} .19)$ \\
$F_{-+\alpha \beta \gamma}$ & $($ C. 30 \\
\hline
\end{tabular}

The fluxes that do not appear in the above tables are not restricted by the Killing spinor equations. $F$ is further restricted by the self-duality condition. The conditions on the geometry have been summarized in section four.

\section{Appendix D The solution of Killing spinor equa- tions for the pure spinor $\eta=1$}

We first begin with the algebraic Killing spinor equations. In particular (3.4) and (3.5) imply that

$$
P_{+}=0, \quad G_{+\alpha}{ }^{\alpha}=0
$$


and (3.6) implies that

$$
G_{+\bar{\alpha} \bar{\beta}}=0 .
$$

The last two equations, (3.2) and (3.3), give

$$
G_{-+\bar{\alpha}}=-G_{\bar{\alpha} \beta}{ }^{\beta}
$$

and

$$
P_{\alpha}+\frac{1}{12} \epsilon_{\alpha}{ }^{\bar{\beta}_{1} \bar{\beta}_{2} \bar{\beta}_{3}} G_{\bar{\beta}_{1} \bar{\beta}_{2} \bar{\beta}_{3}}=0,
$$

respectively.

The trace of the dual of (3.16) gives no new conditions because of (D.1) and the duality condition

$$
F_{+\alpha_{1} \alpha_{2} \alpha_{3} \alpha_{4}}=0 .
$$

Substitute this back into (3.16) to find

$$
G_{+\alpha \bar{\beta}}=0 .
$$

Furthermore (3.10) implies that

$$
F_{+\alpha \bar{\beta} \delta} \delta=-i \Omega_{\alpha,+\bar{\beta}},
$$

the reality condition of $F$ requires that

$$
\Omega_{\alpha,+\bar{\beta}}=-\Omega_{\bar{\beta},+\alpha},
$$

and the duality of $F$ leads to

$$
\Omega_{\alpha,+}{ }^{\alpha}=0 .
$$

The condition (3.24) does not give any additional restrictions on the geometry or the fluxes. Separating (3.22) into real and imaginary pieces, we find that

$$
\partial_{+} \log h+\frac{1}{2} \Omega_{+,-+}=0
$$

and

$$
i \Omega_{+, \gamma}^{\gamma}+Q_{+}=0 .
$$

Next consider the equations (3.11) and (3.15). Dualize (3.11) and symmetrize the free indices to find

$$
\left.F_{+\bar{\gamma}_{1} \bar{\gamma}_{2} \bar{\gamma}_{3}(\alpha} \epsilon_{\beta}\right)^{\bar{\gamma}_{1} \bar{\gamma}_{2} \bar{\gamma}_{3}}=0,
$$

and use the duality of $F$ to get

$$
F_{+\alpha \beta \gamma \bar{\delta}}=0 .
$$


The remaining equation gives

$$
G_{+\alpha \beta}=0
$$

The (3.15) implies

$$
\Omega_{\alpha, \beta+}=0 .
$$

Equation (3.11) gives no new conditions. Next we turn our attention to (3.23). Substituting (D.13) and (D.14), we find that

$$
\Omega_{+, \alpha \beta}=0 .
$$

Taking the real and imaginary parts of (3.17), we find

$$
\partial_{-} \log h+\frac{1}{2} \Omega_{-,-+}=0
$$

and

$$
-i Q_{-}+\Omega_{-, \gamma}^{\gamma}+\frac{i}{2} F_{-\gamma} \gamma^{\delta}=0 .
$$

The latter equation expresses the $F_{-\gamma} \gamma \delta$ component of the flux in terms of the geometry and the scalars. The condition (3.19) gives

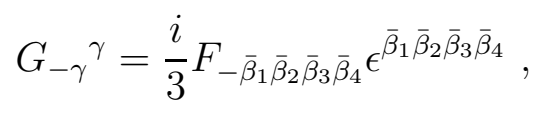

and the condition (3.18) gives

$$
G_{-\alpha \beta}=\epsilon_{\alpha \beta} \bar{\gamma}_{1} \bar{\gamma}_{2}\left[\Omega_{-\bar{\gamma}_{1} \bar{\gamma}_{2}}+i F_{-\bar{\gamma}_{1} \bar{\gamma}_{2} \delta} \delta\right] .
$$

Next let us consider (3.8), (3.12), (3.14) and (3.20). First take the trace of (3.8) to find

$$
\Omega_{\beta,}{ }^{\beta} \bar{\alpha}-i F_{-+\bar{\alpha} \beta}{ }^{\beta}-\frac{1}{8} G_{\gamma_{1} \gamma_{2} \gamma_{3}} \epsilon^{\gamma_{1} \gamma_{2} \gamma_{3}}{ }_{\bar{\alpha}}=0
$$

and (3.201) gives

$$
\Omega_{-,+\bar{\alpha}}+i F_{-+\bar{\alpha} \beta}^{\beta}+\frac{1}{24} G_{\gamma_{1} \gamma_{2} \gamma_{3}} \epsilon^{\gamma_{1} \gamma_{2} \gamma_{3}}{ }_{\bar{\alpha}}=0 .
$$

These give

$$
\Omega_{\beta}{ }^{\beta}{ }_{\bar{\alpha}}+3 \Omega_{-,+\bar{\alpha}}+2 i F_{-+\bar{\alpha} \beta}{ }^{\beta}=0
$$

and

$$
G_{\gamma_{1} \gamma_{2} \gamma_{3}} \epsilon_{{ }_{\bar{\alpha}}}^{\gamma_{1} \gamma_{2} \gamma_{3}}=-24\left[\Omega_{-,+\bar{\alpha}}+i F_{-+\bar{\alpha} \beta}{ }^{\beta}\right]=12\left[\Omega_{-,+\bar{\alpha}}+\Omega_{\beta,}{ }^{\beta} \bar{\alpha}\right] .
$$


Next eliminating $G_{\gamma_{1} \gamma_{2} \gamma_{3}}$ from (3.12), and using (D.21) and (D.24), we get

$$
D_{\bar{\alpha}} \log h+\frac{1}{2} \Omega_{\bar{\alpha}, \beta}{ }^{\beta}+\frac{1}{2} \Omega_{\bar{\alpha},-+}-\frac{1}{4} \Omega_{\beta}{ }^{\beta}{ }_{\bar{\alpha}}-\frac{7}{4} \Omega_{-,+\bar{\alpha}}-\frac{3 i}{2} F_{-+\bar{\alpha} \beta}{ }^{\beta}=0 .
$$

In addition, (3.14) gives

$$
G_{\bar{\alpha} \gamma}^{\gamma}=G_{\bar{\alpha}-+}
$$

Comparing this with (D.3), we find that

$$
G_{\bar{\alpha} \gamma}^{\gamma}=G_{\bar{\alpha}-+}=0
$$

Finally, dualize (3.8) and use (D.24) to find

$$
\left[\Omega_{\alpha, \bar{\gamma}_{1} \bar{\gamma}_{2}}+2 i F_{-+\alpha \bar{\gamma}_{1} \bar{\gamma}_{2}}\right] \epsilon_{\beta_{1} \beta_{2}}^{\bar{\gamma}_{1} \bar{\gamma}_{2}}-2 \Omega_{-,+\bar{\gamma}} \epsilon_{\alpha \beta_{1} \beta_{2}}=0
$$

Dualizing the above equation, we get

$$
\Omega_{\alpha, \bar{\beta}_{1} \bar{\beta}_{2}}+2 i F_{-+\alpha \bar{\beta}_{1} \bar{\beta}_{2}}-2 \Omega_{-,+\left[\bar{\beta}_{1}\right.} g_{\left.\bar{\beta}_{2}\right] \alpha}=0
$$

and taking the trace we find

$$
\Omega_{\beta}{ }^{\beta}{ }_{\bar{\alpha}}+2 i F_{-+\bar{\alpha} \beta}^{\beta}+3 \Omega_{-,+\bar{\alpha}}=0,
$$

which is identical to (D.23).

Next we turn our attention to the remaining four equations (3.7), (3.9), (3.13) and (3.21). First (3.9) implies that

$$
G_{\alpha \gamma}^{\gamma}-G_{-+\alpha}-\frac{2 i}{3} F_{\alpha \bar{\gamma}_{1} \bar{\gamma}_{2} \bar{\gamma}_{3} \bar{\gamma}_{4}} \epsilon^{\bar{\gamma}_{1} \bar{\gamma}_{2} \bar{\gamma}_{3} \bar{\gamma}_{4}}=0 .
$$

Dualizing and taking the trace of (3.13), we get

$$
\frac{1}{2} \Omega_{\bar{\gamma}_{1}, \bar{\gamma}_{2} \bar{\gamma}_{3}} \overline{\bar{\gamma}}^{\bar{\gamma}_{1} \bar{\gamma}_{2} \bar{\gamma}_{3}}{ }_{\alpha}+\frac{1}{8} G_{\alpha \beta}{ }^{\beta}+\frac{3}{8} G_{\alpha-+}=0
$$

and dualizing (3.21), we find

$$
\frac{i}{3} F_{-+\bar{\gamma}_{1} \bar{\gamma}_{2} \bar{\gamma}_{3}} \epsilon^{\bar{\gamma}_{1} \bar{\gamma}_{2} \bar{\gamma}_{3}}{ }_{\alpha}-\frac{1}{8} G_{\alpha \beta}{ }^{\beta}-\frac{3}{8} G_{\alpha-+}=0
$$

Adding the above two equations, we get

$$
3 \Omega_{\left[\bar{\gamma}_{1}, \bar{\gamma}_{2} \bar{\gamma}_{3}\right]}+2 i F_{-+\bar{\gamma}_{1} \bar{\gamma}_{2} \bar{\gamma}_{3}}=0
$$

which expresses $F_{-+\bar{\gamma}_{1} \bar{\gamma}_{2} \bar{\gamma}_{3}}$ in terms of the geometry. In addition comparing (D.31) and (D.33), and using the duality of $F$, we get

$$
G_{\alpha-+}=0
$$


and

$$
G_{\alpha \beta}{ }^{\beta}=\frac{8 i}{3} F_{-+\bar{\gamma}_{1} \bar{\gamma}_{2} \bar{\gamma}_{3}} \epsilon^{\bar{\gamma}_{1} \bar{\gamma}_{2} \bar{\gamma}_{3}}{ }_{\alpha}=-4 i \Omega_{\gamma_{1}, \bar{\gamma}_{2} \bar{\gamma}_{3}} \epsilon^{\bar{\gamma}_{1} \bar{\gamma}_{2} \bar{\gamma}_{3}}{ }_{\alpha}
$$

By substituting the above results into (3.13), we also find

$$
G_{\gamma_{1} \gamma_{2} \bar{\alpha}}=\left(\Omega_{\bar{\alpha}, \bar{\delta}_{1} \bar{\delta}_{2}}-2 i F_{-+\bar{\alpha} \bar{\delta}_{1} \bar{\delta}_{2}}\right) \epsilon^{\bar{\delta}_{1} \bar{\delta}_{2}} \gamma_{1} \gamma_{2}=2\left[\Omega_{\bar{\alpha}, \bar{\delta}_{1} \bar{\delta}_{2}}+\Omega_{\bar{\delta}_{1}, \bar{\delta}_{2} \bar{\alpha}}\right] \epsilon^{\bar{\delta}_{1} \bar{\delta}_{2}} \gamma_{1} \gamma_{2} .
$$

Finally taking the complex conjugate of (3.7), we get

$$
D_{\bar{\alpha}}^{*} \log h-\frac{1}{2} \Omega_{\bar{\alpha}, \beta}{ }^{\beta}+\frac{1}{2} \Omega_{\bar{\alpha},-+}+\frac{1}{4} \Omega_{\beta}{ }^{\beta}{ }_{\bar{\alpha}}+\frac{3}{4} \Omega_{-,+\bar{\alpha}}+\frac{3 i}{2} F_{-+\bar{\alpha} \beta}{ }^{\beta}=0
$$

Comparing this with (D.25) we find

$$
2 \partial_{\bar{\alpha}} \log h-\Omega_{\bar{\alpha},+-}-\Omega_{-,+\bar{\alpha}}=0
$$

and

$$
\begin{aligned}
i Q_{\bar{\alpha}}-\Omega_{\bar{\alpha}, \beta}{ }^{\beta}+\frac{1}{2} \Omega_{\beta},_{\bar{\alpha}} & +\frac{5}{2} \Omega_{-,+\bar{\alpha}} \\
+3 i F_{-+\bar{\alpha} \gamma}{ }^{\gamma} & =i Q_{\bar{\alpha}}-\Omega_{\bar{\alpha}, \beta}{ }^{\beta}-\Omega_{\beta,{ }_{\bar{\alpha}}{ }^{\beta}-2 \Omega_{-,+\bar{\alpha}}=0 .}
\end{aligned}
$$

The scalar fluxes $P$ are given in the following equations

\begin{tabular}{|c|c|}
\hline Fluxes & Equations \\
\hline$P_{+}$ & $(\mathrm{D} .1)$ \\
$P_{\alpha}$ & $(\mathrm{D} .4$ \\
$P_{\bar{\alpha}}$ & $($ 3.2) \\
\hline
\end{tabular}

The $G$ fluxes are determined by the following equations

\begin{tabular}{|c|c|}
\hline Fluxes & Equations \\
\hline$G_{+\alpha}^{\alpha}$ & $(\mathrm{D} .1)$ \\
$G_{+\alpha \bar{\beta}}$ & $(\mathrm{D} .6)$ \\
$G_{+\alpha \beta}$ & $(\mathrm{D} .14$ \\
$G_{+\bar{\alpha} \bar{\beta}}$ & $(\mathrm{D} .2)$ \\
$G_{-\alpha}^{\alpha}$ & $(\mathrm{D} .19$ \\
$G_{-\alpha \beta}$ & $(\mathrm{D} .20$ \\
$G_{\alpha \beta \gamma}$ & $(\mathrm{D} .24$ \\
$G_{-+\bar{\alpha}}$ & $(\mathrm{D} .27$ \\
$G_{-+\alpha}$ & $(\mathrm{D} .35$ \\
$G_{\alpha \beta \bar{\gamma}}$ & $(\mathrm{D} .37$ \\
\hline
\end{tabular}

The $F$ fluxes are determined by the following equations

\begin{tabular}{|l|c|}
\hline Fluxes & Equations \\
\hline$F_{+\alpha \beta \gamma \delta}$ & $(\mathrm{B} .4)$ \\
$F_{+\alpha \bar{\beta} \gamma} \gamma$ & $(\mathrm{D} .7$ \\
$F_{+\alpha \beta \gamma \bar{\delta}}$ & $(\mathrm{D} .13$ \\
$F_{-\alpha}{ }^{\alpha} \beta$ & $(\mathrm{D} .18$ \\
$F_{-+\alpha \beta \gamma}$ & $(\mathrm{D} .34$ \\
$F_{-+\alpha \bar{\beta} \bar{\gamma}}$ & $(\mathrm{D} .29$ \\
\hline
\end{tabular}


The fluxes that do not appear in the above tables are not restricted by the Killing spinor equations. $F$ is further restricted by the self-duality condition. The conditions on the geometry are summarized in section 5 .

\section{Appendix E The solution of Killing spinor equa- tions for the pure spinor $\eta=e_{1234}$}

We first begin with the algebraic Killing spinor equations. In particular (3.4) and (3.5) imply that

$$
P_{+}=0, \quad G_{+\alpha}{ }^{\alpha}=0
$$

and (3.6) implies that

$$
G_{+\alpha \beta}=0 .
$$

The last two equations, (3.2) and (3.3), give

$$
G_{-+\alpha}=G_{\alpha \beta} \beta
$$

and

$$
P_{\bar{\alpha}}+\frac{1}{12} \epsilon_{\bar{\alpha}}^{\beta_{1} \beta_{2} \beta_{3}} G_{\beta_{1} \beta_{2} \beta_{3}}=0,
$$

respectively.

The trace of the dual of (3.10) gives the duality condition

$$
F_{+\alpha_{1} \alpha_{2} \alpha_{3} \alpha_{4}}=0 .
$$

Substitute this back into (3.10) to find

$$
G_{+\alpha \bar{\beta}}=0 .
$$

Furthermore (3.16) implies that

$$
F_{+\alpha \bar{\beta} \delta} \delta=i \Omega_{\alpha,+\bar{\beta}},
$$

the reality condition of $F$ requires that

$$
\Omega_{\alpha,+\bar{\beta}}=-\Omega_{\bar{\beta},+\alpha},
$$

and the duality condition of $F$ gives

$$
\Omega_{\alpha,+}{ }^{\alpha}=0 .
$$

The condition (3.22) does not give any additional restrictions on the geometry or the fluxes. Separating (3.24) into real and imaginary pieces, we find that

$$
\partial_{+} \log h+\frac{1}{2} \Omega_{+,-+}=0
$$


and

$$
-i \Omega_{+, \gamma}^{\gamma}+Q_{+}=0
$$

Next consider the equations (3.11), (3.15) and use the duality of $F$. They yield

$$
\begin{gathered}
F_{+\alpha \beta \gamma \bar{\delta}}=0, \\
\Omega_{\bar{\alpha}, \bar{\beta}+}=0,
\end{gathered}
$$

and

$$
G_{+\bar{\alpha} \bar{\beta}}=0 .
$$

Next we turn our attention to (3.23). Using the above results, we find that

$$
\Omega_{+, \alpha \beta}=0 \text {. }
$$

Taking the real and imaginary parts of (3.19), we find

$$
\partial_{-} \log h+\frac{1}{2} \Omega_{-,-+}=0
$$

and

$$
-i Q_{-}-\Omega_{-, \gamma}^{\gamma}+\frac{i}{2} F_{-\gamma}{ }^{\gamma} \delta=0
$$

The latter equation can expresses the $F_{-\gamma}{ }^{\gamma} \delta$ component of the flux in terms of the geometry and the scalars. The condition (3.17) gives

$$
G_{-\gamma}^{\gamma}=-\frac{i}{3} F_{-\beta_{1} \beta_{2} \beta_{3} \beta_{4}} \epsilon^{\beta_{1} \beta_{2} \beta_{3} \beta_{4}}
$$

and the condition (3.18) gives

$$
G_{-\bar{\alpha} \bar{\beta}}=\epsilon_{\bar{\alpha} \bar{\beta}}^{\gamma_{1} \gamma_{2}}\left[\Omega_{-, \gamma_{1} \gamma_{2}}-i F_{-\gamma_{1} \gamma_{2} \delta} \delta^{\delta}\right] .
$$

Next let us consider (3.7), (3.9), (3.13) and (3.21). An analysis similar to the one in the previous appendix yields

$$
\Omega_{\beta}{ }^{\beta} \bar{\alpha}+3 \Omega_{-,+\bar{\alpha}}-2 i F_{-+\bar{\alpha} \beta}^{\beta}=0
$$

and

$$
G_{\bar{\gamma}_{1} \bar{\gamma}_{2} \bar{\gamma}_{3}} \epsilon^{\bar{\gamma}_{1} \bar{\gamma}_{2} \bar{\gamma}_{3}}=-24\left[\Omega_{-,+\alpha}-i F_{-+\alpha \beta}{ }^{\beta}\right]=12\left[\Omega_{-,+\alpha}+\Omega_{\bar{\beta},{ }_{\alpha}{ }^{\bar{\beta}}}\right]
$$

The former condition has been viewed as an equation for $F_{-+\bar{\alpha} \beta} \beta$. By substituting these results back into the equations we find

$$
D_{\bar{\alpha}}^{*} \log h+\frac{1}{2} \Omega_{\bar{\alpha}, \beta}{ }^{\beta}+\frac{1}{2} \Omega_{\bar{\alpha},-+}-\frac{1}{4} \Omega_{\beta}{ }^{\beta}{ }_{\bar{\alpha}}-\frac{7}{4} \Omega_{-,+\bar{\alpha}}+\frac{3 i}{2} F_{-+\bar{\alpha} \beta}{ }^{\beta}=0 .
$$


and

$$
G_{\alpha \gamma}^{\gamma}=G_{\alpha-+}=0
$$

Finally, dualizing (3.13) yields

$$
\Omega_{\bar{\alpha}, \beta_{1} \beta_{2}}+2 i F_{-+\bar{\alpha} \beta_{1} \beta_{2}}-2 \Omega_{-,+\left[\beta_{1}\right.} g_{\left.\beta_{2}\right] \bar{\alpha}}=0 .
$$

Next we turn our attention to the remaining four equations (3.8), (3.12), (3.14) and (3.20). They yield

$$
3 \Omega_{\left[\bar{\gamma}_{1}, \bar{\gamma}_{2} \bar{\gamma}_{3}\right]}-2 i F_{-+\bar{\gamma}_{1} \bar{\gamma}_{2} \bar{\gamma}_{3}}=0
$$

which expresses $F_{\bar{\gamma}_{1} \bar{\gamma}_{2} \bar{\gamma}_{3} \delta} \delta$ in terms of the other fluxes and geometry. We also find that

$$
G_{\bar{\alpha}-+}=0
$$

and

$$
G_{\bar{\alpha} \beta}^{\beta}=-\frac{8 i}{3} F_{-+\gamma_{1} \gamma_{2} \gamma_{3}} \epsilon_{\bar{\alpha}}^{\gamma_{1} \gamma_{2} \gamma_{3}}=4 \Omega_{\gamma_{1}, \gamma_{2} \gamma_{3}} \epsilon^{\gamma_{1} \gamma_{2} \gamma_{3}}{ }_{\bar{\alpha}}
$$

By substituting the above results into (3.8) we get

$$
\begin{aligned}
G_{\alpha \bar{\gamma}_{1} \bar{\gamma}_{2}} & =\left(\Omega_{\alpha, \delta_{1} \delta_{2}}-2 i F_{-+\alpha \delta_{1} \delta_{2}}\right) \epsilon^{\delta_{1} \delta_{2}} \bar{\gamma}_{1} \bar{\gamma}_{2} \\
& =2\left[\Omega_{\alpha, \delta_{1} \delta_{2}}+\Omega_{\delta_{1}, \delta_{2} \alpha}\right] \epsilon^{\delta_{1} \delta_{2}} \bar{\gamma}_{1} \bar{\gamma}_{2} .
\end{aligned}
$$

Finally, comparing (3.14) with (E.22) yields

$$
2 \partial_{\bar{\alpha}} \log h-\Omega_{\bar{\alpha},+-}-\Omega_{-,+\bar{\alpha}}=0
$$

and

$$
\begin{aligned}
-i Q_{\bar{\alpha}}-\Omega_{\bar{\alpha}, \beta}{ }^{\beta}+\frac{1}{2} \Omega_{\beta}{ }^{\beta}{ }_{\bar{\alpha}} & +\frac{5}{2} \Omega_{-,+\bar{\alpha}} \\
-3 i F_{-+\bar{\alpha} \gamma}{ }^{\gamma} & =-i Q_{\bar{\alpha}}-\Omega_{\bar{\alpha}, \beta}{ }^{\beta}-\Omega_{\beta}{ }^{\beta}{ }_{\bar{\alpha}}-2 \Omega_{-,+\bar{\alpha}}=0 .
\end{aligned}
$$

This concludes the analysis of the equations. The conditions on the geometry are summarized in section 5 .

\section{References}

[1] J. Figueroa-O'Farrill and G. Papadopoulos, "Maximally supersymmetric solutions of ten-dimensional and eleven-dimensional supergravities," JHEP 0303 (2003) 048: arXiv:hep-th/0211089.

[2] J. Figueroa-O'Farrill and G. Papadopoulos, "Pluecker-type relations for orthogonal planes," arXiv:math.ag/0211170.

[3] J. P. Gauntlett and S. Pakis, "The geometry of D = 11 Killing spinors," JHEP 0304 (2003) 039 arXiv:hep-th/0212008. 
[4] J. P. Gauntlett, J. B. Gutowski and S. Pakis, "The geometry of D = 11 null Killing spinors," JHEP 0312 (2003) 049 arXiv:hep-th/0311112.

[5] M. J. Duff and J. T. Liu, "Hidden spacetime symmetries and generalized holonomy in M-theory," Nucl. Phys. B 674 (2003) 217 arXiv:hep-th/0303140.

[6] C. Hull, "Holonomy and symmetry in M-theory," arXiv:hep-th/0305039.

[7] G. Papadopoulos and D. Tsimpis, "The holonomy of the supercovariant connection and Killing spinors," JHEP 0307 (2003) 018 arXiv:hep-th/0306117.

[8] I. A. Bandos, J. A. de Azcarraga, J. M. Izquierdo, M. Picon and O. Varela, "On BPS preons, generalized holonomies and D = 11 supergravities," Phys. Rev. D 69 (2004) 105010 arXiv:hep-th/0312266.

[9] A. Batrachenko, J. T. Liu, O. Varela and W. Y. Wen, "Higher order integrability in generalized holonomy," arXiv:hep-th/0412154

[10] J. Figueroa-O'Farrill, P. Meessen and S. Philip, "Supersymmetry and homogeneity of M-theory backgrounds," Class. Quant. Grav. 22 (2005) 207 arXiv:hep-th/0409170.

[11] J. Gillard, U. Gran and G. Papadopoulos, "The spinorial geometry of supersymmetric backgrounds," arXiv:hep-th/0410155

[12] J. H. Schwarz, "Covariant Field Equations Of Chiral N=2 D = 10 Supergravity," Nucl. Phys. B 226 (1983) 269.

[13] P. S. Howe and P. C. West, "The Complete N=2, D = 10 Supergravity," Nucl. Phys. B 238 (1984) 181.

[14] M. Blau, J. Figueroa-O'Farrill, C. Hull and G. Papadopoulos, "A new maximally supersymmetric background of IIB superstring theory," JHEP 0201 (2002) 047 arXiv:hep-th/0110242.

[15] M. Blau, J. Figueroa-O'Farrill, C. Hull and G. Papadopoulos, "Penrose limits and maximal supersymmetry," Class. Quant. Grav. 19 (2002) L87 arXiv:hep-th/0201081.

[16] G. Papadopoulos and D. Tsimpis, "The holonomy of IIB supercovariant connection," Class. Quant. Grav. 20 (2003) L253 arXiv:hep-th/0307127.

[17] E. J. Hackett-Jones and D. J. Smith, "Type IIB Killing spinors and calibrations," JHEP 0411 (2004) 029 arXiv:hep-th/0405098.

[18] K. Pilch and N. P. Warner, "N = 1 supersymmetric solutions of IIB supergravity from Killing spinors," arXiv:hep-th/0403005.

[19] J. H. Schwarz and P. C. West, "Symmetries And Transformations Of Chiral N=2 D = 10 Supergravity," Phys. Lett. B 126 (1983) 301.

[20] M. Cederwall, U. Gran, M. Nielsen and B. E. W. Nilsson, "(p,q) 5-branes in non-zero B-field," JHEP 0001 (2000) 037 arXiv:hep-th/9912106.

[21] U. Gran, J. Gutowski and G. Papadopoulos, in preparation.

[22] M. Grana, R. Minasian, M. Petrini and A. Tomasiello, "Supersymmetric backgrounds from generalized Calabi-Yau manifolds," JHEP 0408 (2004) 046 arXiv:hep-th/0406137.

A. Butti, M. Grana, R. Minasian, M. Petrini and A. Zaffaroni, "The baryonic branch of Klebanov-Strassler solution: A supersymmetric family of SU(3) structure backgrounds," arXiv:hep-th/0412187. 
[23] J. M. Figueroa-O'Farrill, "Breaking the M-waves," Class. Quant. Grav. 17 (2000) 2925 arXiv:hep-th/9904124.

[24] R. Bryant, "Pseudo-Riemannian metrics with parallel spinor fields and vanishing Ricci tensor," arXiv:math.DG/0004073.

[25] R. Hernandez, K. Sfetsos and D. Zoakos, "Supersymmetry and Lorentzian holonomy in various dimensions," JHEP 0409 (2004) 010 arXiv:hep-th/0406105.

[26] S. Salamon, "Riemannian Geometry and Holonomy Groups," Pitman Research Notes in Mathematics Series, Longman Group UK (1989).

[27] A. Moroianu and U. Semmelmann, "Parallel spinors and holonomy group," math.DG/9903062.

[28] B. McInnes, Spin holonomy of Einstein manifolds, Commun. Math. Phys. 203 (1999) 349-364.

[29] K. Becker and L. S. Tseng, "A note on fluxes in six-dimensional string theory backgrounds," arXiv:hep-th/0410283.

[30] R. Penrose, "Any spacetime has a plane wave as a limit," Differential Geometry and Relativity, 271-275, Riedel Dordrecht, (1976).

[31] M. Blau, J. Figueroa-O'Farrill and G. Papadopoulos, "Penrose limits, supergravity and brane dynamics," Class. Quant. Grav. 19 (2002) 4753 arXiv:hep-th/0202111.

[32] A. Gray and L.M. Hervella, "The sixteen classes of almost Hermitian manifolds and their linear invariants", Ann. Mat. Pura e Appl. 282 (1980) 1.

[33] F. R. Harvey, "Spinors and Calibrations," Academic Press, London (1990).

[34] J. Gutowski and G. Papadopoulos, "AdS calibrations," Phys. Lett. B 462 (1999) 81 arXiv:hep-th/9902034.

[35] J. Gutowski, G. Papadopoulos and P. K. Townsend, "Supersymmetry and generalized calibrations," Phys. Rev. D 60 (1999) 106006 arXiv:hep-th/9905156.

[36] M. Fernandez, "A classification of Riemannian manifolds with structure Spin(7)," Ann. Mat. Pura Appl. 143 (1982) 101.

[37] S. Chiossi and S. Salamon, "The intrinsic torsion of $S U(3)$ and $G_{2}$ structures", Diff. Geom., Valencia 2001, World Sci. 2002, 115 arXiv:math.DG/0202282.

[38] F. M. Cabrera, "Special almost Hermitian geometry," math.DG/0409167

[39] S. Ivanov, "Connection with torsion, parallel spinors and geometry of Spin(7) manifolds," Math. Res. Lett. 11 (2004), no. 2-3, 171-186, math.DG/0111216.

[40] H. Blaine Lawson and Marie-Louise Michelsohn, "Spin geometry," Princeton University Press (1989).

[41] McKenzie Y. Wang, "Parallel spinors and parallel forms", Ann. Global Anal Geom. Vol 7, No 1 (1989), 59.

[42] O. A. P. Mac Conamhna, "Refining G-structure classifications," arXiv:hep-th/0408203. 